\title{
Natural Laminar Flow Design for Wings with Moderate Sweep
}

\author{
Richard L. Campbell ${ }^{1}$ and Michelle N. Lynde ${ }^{2}$ \\ NASA Langley Research Center, Hampton, VA, 23681
}

\begin{abstract}
A new method for the aerodynamic design of wings with natural laminar flow is under development at the NASA Langley Research Center. The approach involves the addition of new flow constraints to an existing knowledge-based design module for use with advanced flow solvers. The uniqueness of the new approach lies in the tailoring of target pressure distributions to achieve laminar flow on transonic wings with leading-edge sweeps and Reynolds numbers typical of current transports. The method is demonstrated on the Common Research Model configuration at critical $\mathrm{N}$-factor levels representative of both flight and high-Reynolds number wind tunnel turbulence levels. The design results for the flight conditions matched the target extent of laminar flow very well. The design at wind tunnel conditions raised some design issues that prompted further improvements in the method, but overall has given promising results.
\end{abstract}

\section{Nomenclature}

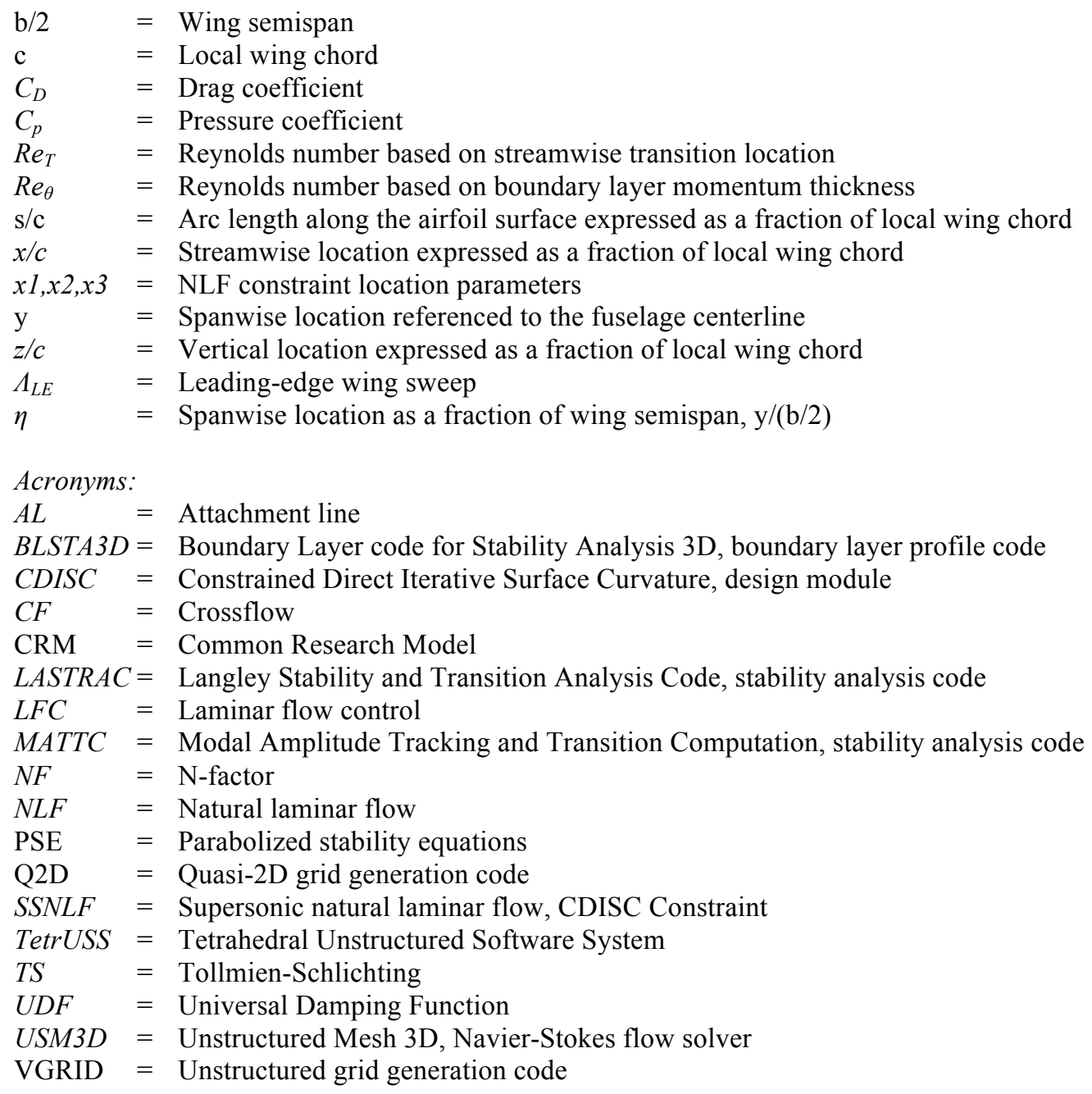

${ }^{1}$ Senior Research Engineer, Configuration Aerodynamics Branch, NASA Langley Research Center M/S 499 Hampton, VA 23681, and AIAA Associate Fellow.

${ }^{2}$ Research Aerospace Engineer, Configuration Aerodynamics Branch, NASA Langley Research Center M/S 499 Hampton, VA 23681, and AIAA Member. 


\section{Introduction}

$\mathrm{A}$ $\mathrm{s}$ the cost of fuel continues to increase over time and concern for the environment drives acceptable levels of emissions lower, the goal of reducing drag and the associated fuel burn becomes increasingly important. One of the key technologies for reducing drag identified in many studies is laminar flow. Although this technology was first demonstrated on aircraft several decades ago ${ }^{1}$, it is only recently that it has begun to be used on commercial transports $^{2}$. In a recent Technical Interchange Meeting at NASA Langley on the future of Green Aviation, laminar flow on wings at high Reynolds number was identified as the "final challenge" relative to laminar flow applications on commercial aircraft ${ }^{3}$.

One reason for the limited application of laminar flow is that, for most modern transports, the wing and other similar components are swept to allow higher cruise speeds. This sweep has an adverse effect on the growth of crossflow $^{1}$ (CF) disturbances in the boundary layer near the leading edge that can greatly reduce the extent of laminar flow obtained. Historically, the approaches used to control CF growth have been to limit the leading-edge sweep to about 20 degrees or less, or to employ suction through a porous or slotted surface near the leading edge.

The first approach retains the simplicity of natural laminar flow (NLF) in that no ducting, special material or suction source is required, but it also tends to limit the cruise speed of the vehicle. Seitz ${ }^{4}$ proposes an interesting variation of this approach by using a forward-swept, tapered wing that has the low leading-edge sweep required to avoid $\mathrm{CF}$ growth, but has the same sweep at the mid-chord shock location, and thus potentially similar wave drag levels, as a typical aft-swept wing. As pointed out by Seitz, other issues, such as wing static-aeroelastic divergence and flap effectiveness reduction due to higher trailing-edge sweep, would have to be considered in the trades between such a configuration and an aft-swept one.

The second approach, using suction near the leading edge and relying on NLF pressure distribution tailoring to control Tollmien-Schlichting (TS) disturbance growth in the mid-chord region, is referred to as hybrid natural laminar flow (HNLF), a subset of laminar flow control (LFC). The power required for the suction, along with the additional manufacturing and weight penalties, tend to reduce the case for utilizing this approach. Boeing has developed an innovative variation of this approach for the vertical tail of their 787-9 aircraft that utilizes a passive system to provide the needed suction ${ }^{2}$.

A third approach, distributed roughness elements or DREs, has been proposed by Saric ${ }^{5-6}$. This passive method positions disturbance-creating elements near the wing leading edge with periodic spanwise spacing selected to excite noncritical CF wavelengths, altering the mean flow and reducing the energy available for growth of what would have been critical wavelengths. While early results at lower Reynolds numbers appeared promising, more recent flight tests have provided inconsistent results and further research is needed.

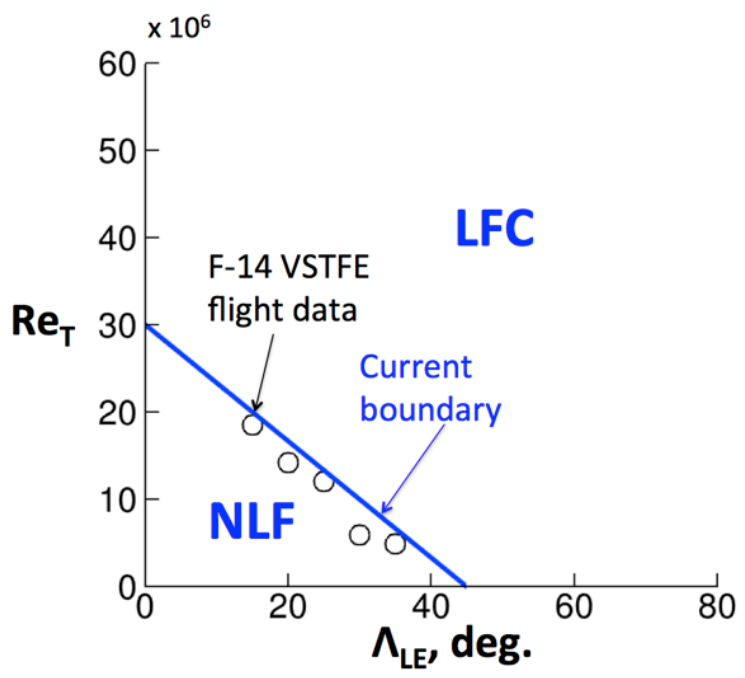

Figure 1 - Current NLF-LFC boundary in terms of leading-edge sweep $\left(\Lambda_{\mathrm{LE}}\right)$ and transition Reynolds number $\left(\operatorname{Re}_{\mathrm{T}}\right)$.

Figure 1 shows an approximate limiting boundary between NLF and LFC in terms of leading-edge sweep and transition Reynolds number. The boundary line is a linear approximation to the highest values obtained during the F14 Variable Sweep Transition Flight Experiment ${ }^{7}$, which represent an upper bound to other flight and wind tunnel 
data for NLF. Based on this figure, it is easy to see why the current and near-term NLF applications are on nacelles (with essentially no sweep) and winglets that have sweep but small chords and thus low Reynolds numbers.

Our new NLF design method proposes that it may be possible to expand the current boundary for NLF through careful tailoring of the pressure distribution near the leading edge of a wing or similar component. This general type of NLF target pressure was first used by the authors in the Automated Target Pressure Generation method ${ }^{8}$ developed during a cooperative effort between researchers from NASA and the DLR in Braunschweig, Germany. The motivation to apply it to wings with moderate-to-high sweep, however, came from a similar cooperative effort with researchers from JAXA in Japan, who had developed a similar pressure architecture using their own methodology and were already applying it to supersonic wings ${ }^{9-10}$. The initial work in extending our NLF design method for application to wings with higher sweep was done in this same context and is included in a companion paper entitled "Expanding the Natural Laminar Flow Boundary for Supersonic Transports". The current paper describes the modification and application of this approach to transonic transports.

\section{NLF Design Approach}

In the following sections, the current status of our transonic NLF design efforts will be described in an attempt to answer the following three questions:

1) Can a target pressure distribution be defined that will support a significant region of laminar flow and meet other flow constraints (lift, pitching moment, shock strength, etc.)?

2) Can a geometry be designed to match the target pressures with sufficient accuracy to largely obtain the target laminar flow region while matching geometry constraints (thickness, leading-edge radius, twist, etc.)?

3) Do the attachment line characteristics for the design fall within the required limits to avoid attachment line contamination/transition?

Even if all of the above are confirmed, questions relating to the practicality of the resulting wing design, such as the cost of manufacturing and maintaining the wing as well as any adverse impact at the off-design conditions, would need to be answered. Our current work does not address these concerns other than that we are limiting our laminar flow region to the upper surface of the wing due to the assumption of a Krueger flap for insect protection as well as avoiding gaps or steps in the laminar region.

\section{Methods}

Our proposed NLF design method utilizes new flow and geometry constraints in the CDISC knowledge-based design method ${ }^{8,11}$ within the NLF analysis and design framework shown in Figure 2. The methods highlighted in blue were used in the current study, with details on the codes and options selected given below.

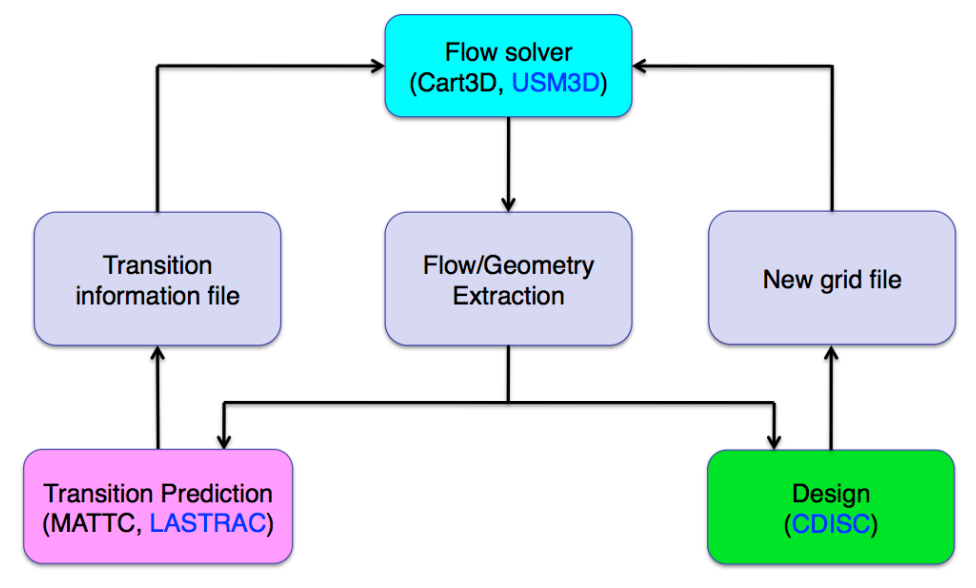

Figure 2 - Flow chart of the NLF design and analysis framework. 


\section{A. Grid generation and flow solution}

The TetrUSS software system ${ }^{12}$ was used to generate the grids and flow solutions for the cases in this study. The VGRID code ${ }^{13}$ generates a triangular surface mesh and then builds viscous grid cells using an advancing layers method. The remaining inviscid portion of the grid is filled using an advancing front method. All of the cells in these unstructured grids are tetrahedrons with $\mathrm{y}+=0.5$ for the first cell center off of the surface.

The flow solutions were obtained using the USM $3 \mathrm{D}^{14}$ component of the TetrUSS system. This code is an upwind, finite-volume Navier-Stokes flow solver that uses flux-difference splitting to compute the inviscid flux quantities across cell faces. For the cases shown in this paper, the Spalart-Allmaras turbulence model ${ }^{15}$ was used in regions of turbulent flow while the forced-laminarization option ${ }^{16}$ was used ahead of the predicted transition location on the wing.

\section{B. Boundary layer stability analysis}

Two stability analysis methods are available to predict the growth of TS and CF mode curves. The MATTC $\operatorname{code}^{8}$ is an empirical method that is very efficient and reasonably accurate, especially when calibrated using results from a higher-order method such as LASTRAC ${ }^{17}$. MATTC computes the $\mathrm{N}$-factor growth curves directly from the pressure distributions at the wing design stations and thus does not need boundary layer profiles extracted from the flow solution or generated by an auxiliary boundary layer solver. For the unique pressure distributions used in this study, however, there was some question as to how well the MATTC empirical model would work, so it was decided to use the linear stability theory option in LASTRAC for the stability analyses.

The LASTRAC code is actually a collection of methods ranging in fidelity from linear stability theory to linear/nonlinear parabolized stability equations (PSE) and secondary instability theory. LASTRAC has been used for $\mathrm{N}$-factor correlation studies (including the development of empirical methods), absolute-amplitude transition analysis and receptivity computations and is applicable in all speed regimes. For consistency with our supersonic work and to save on computational resources, it was decided to use the BLSTA3D boundary layer code ${ }^{18}$ and the extracted streamwise pressure distributions to provide the boundary layer profile input required by LASTRAC.

\section{Design}

The CDISC module is a knowledge-based design method that has been coupled with a variety of 2-D and 3-D flow solvers with physics models ranging from potential flow to Navier-Stokes. The code uses specified flow/geometry relationships developed from analytical or empirical studies to compute geometry changes based on the difference between current and target flow quantities. This eliminates the need to compute sensitivity derivatives and allows the design to converge in parallel with the flow solution, thus greatly reducing the time required for a design. The design time is further reduced by the use of flow constraints to automatically develop the target distribution from the current values of flow quantities such as pressure or skin-friction coefficients. In addition to the flow constraints, geometry constraints such as thickness, curvature, volume and leading-edge radius are available to address requirements from other disciplines such as structures and manufacturing. The development of a new flow constraint to address an NLF design is described in the following section.

\section{CDISC NLF Flow Constraint}

A description of the rationale behind our new NLF flow constraint is provided in the following paragraphs. While the original development of this approach was done in the context of supersonic transports, the description below only addresses the issues related to transonic flow.

For transonic wings with leading-edge sweeps greater than about 20 degrees, there are three primary causes of transition that need to be addressed in any NLF design approach. The first of these, attachment line (AL) contamination/transition, has the potential to completely eliminate laminar flow on the wing. This form of transition is addressed in our method by imposing a wing leading-edge radius constraint based on Poll's criteria ${ }^{19}$ and can lead to fairly small radii for airfoil leading-edge geometries for higher sweeps and Reynolds numbers. If contamination of the attachment line by the turbulent fuselage boundary layer can be avoided by using a device such as a Gaster bump $^{20}$, a less stringent criteria for suppressing attachment line transition can be used, resulting in an increase of at least a factor of four in the maximum allowable leading-edge radius value. The effect of this constraint will be illustrated in the wing design example included in the V. Results section later in this paper.

The other two primary forms of transition, Tollmien-Schlicting (TS) and crossflow (CF) instabilities, are addressed in the design process by the development of a new CDISC flow constraint that will automatically generate 
a target pressure distribution that suppresses the growth of these modes. The basic architecture of this pressure distribution is shown in Figure 3. The strategy for determining the streamwise location nondimensionalized by local chord $(\mathrm{x} / \mathrm{c})$ of the three break points $\mathrm{x} 1, \mathrm{x} 2$ and $\mathrm{x} 3$, as well as their pressure coefficient $\left(\mathrm{C}_{\mathrm{p}}\right)$ values, will be described below. As noted above, for all of the examples given in this paper, NLF is only considered on the upper surface of a wing.

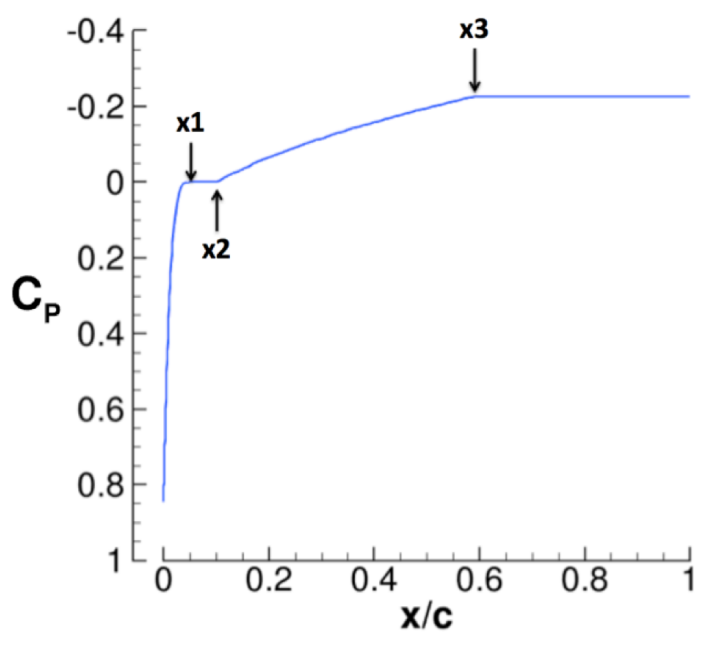

Figure 3 - NLF target pressure architecture.

The second most critical mode of transition after AL in terms of early loss of laminar flow is CF. The strong favorable gradient near the leading edge causes a rapid growth in the CF instabilities that for typical current transport wings would lead to transition within the first 5-10 percent of the airfoil surface. To illustrate this, a code that generates generic target pressure distributions was used to create one having an attachment line $\mathrm{C}_{\mathrm{p}}$ corresponding to a wing with 30 degrees of sweep, then accelerating to $C_{p}=0$ at $x 1$ and remaining at that value to the end of the airfoil, simulating a swept flat plate. This target pressure was run in the BLSTA3D code at a chord Reynolds number of 30 million with the resulting boundary layer analyzed in LASTRAC for CF growth. The results are plotted in Figure 4, with the blue curves showing the growth of disturbances at the input wave numbers and the dashed red line representing an envelope of the highest values obtained at each $\mathrm{x} / \mathrm{c}$. As seen in this figure, the peak $\mathrm{N}$-factor (NF) near the leading edge is greater than 14. If a critical $\mathrm{N}$-factor level of 9 (black horizontal line) is assumed, transition would occur in the first few percent chord.

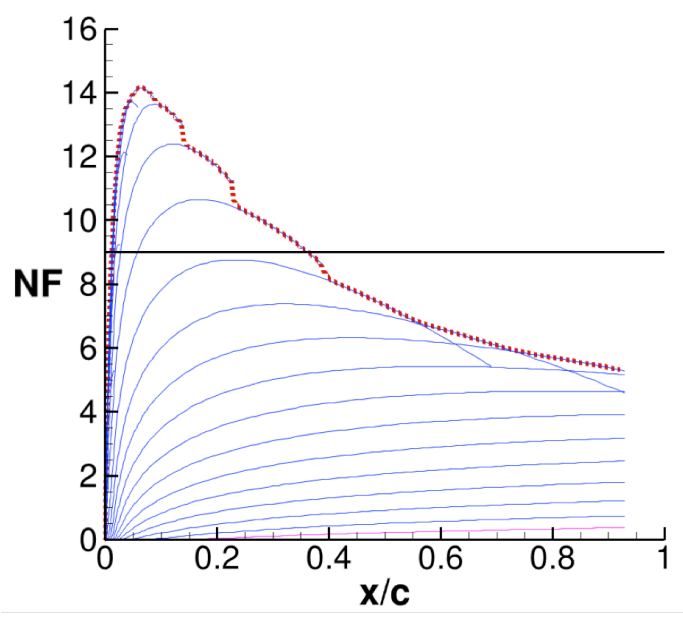

Figure 4 - LASTRAC CF analysis for simulated flat plate.

American Institute of Aeronautics and Astronautics 
The target pressure generation code was then used to create similar cases with differing values of $\mathrm{x} 1$. The resulting peak $\mathrm{N}$-factors for each value of $\mathrm{x} 1$ are plotted in Figure 5, where $\mathrm{x} 1$ corresponds to the arc length (s/c) along the airfoil surface from the attachment line. The results show a fairly linear relationship that can be used to determine a value of $\mathrm{x} 1$ that will keep the $\mathrm{CF} \mathrm{N}$-factor peak below a given critical level. This approach was further calibrated to account for variations in leading-edge sweep and Reynolds number and incorporated into the new CDISC flow constraint.

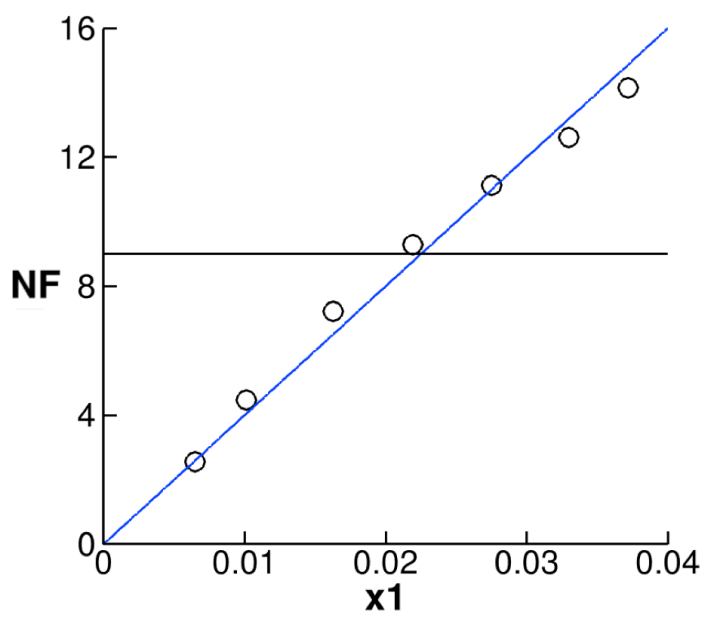

Figure 5 - Effect of the $x 1$ parameter on peak CF N-factor.

Based on Figure 5, a value of $\mathrm{x} 1=0.016$ was selected to provide some margin from the critical $\mathrm{N}$-factor for $\mathrm{CF}$ (see Figure 6b), then this target pressure was also analyzed in LASTRAC for TS growth, with the TS results shown in Figure 6a, where the blue curves represent individual frequencies. The flat pressure distribution aft of x1 produces a strong growth in the TS instabilities that would cause transition at about $\mathrm{x} / \mathrm{c}=0.2$ for the assumed critical $\mathrm{N}$-factor of 9. In order to suppress the TS growth, a favorable pressure gradient can be applied starting at x2 and ending at x3, with a flat pressure gradient retained between $\mathrm{x} 1$ and $\mathrm{x} 2$ to ensure suppression of the CF growth as will be illustrated below.

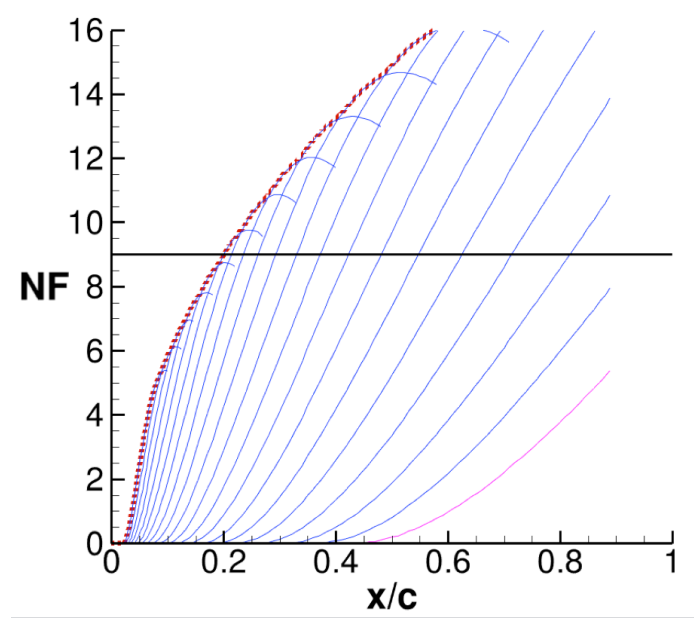

a) TS.

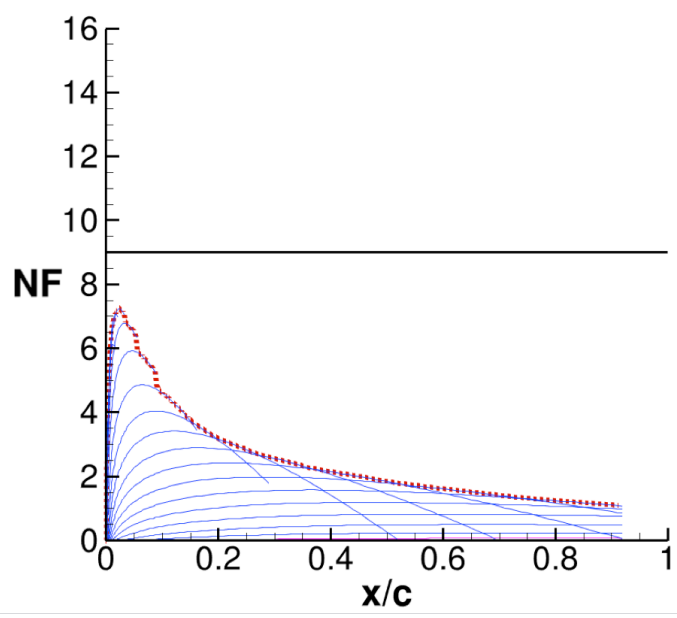

b) $\mathrm{CF}$.

Figure 6 - LASTRAC analysis of flat plate target pressures for $\mathbf{x 1}=\mathbf{0 . 0 1 6}$. 
Several methods for prescribing the $\mathrm{C}_{\mathrm{p}}$ values between $\mathrm{x} 2$ and $\mathrm{x} 3$ were tried. The final one selected is referred to as the Universal Damping Function (UDF), in part because of its ability to completely flatten TS growth at a desired $\mathrm{N}$-factor level with the specification of a single parameter. Figure 6a illustrates the UDF applied to the previous case at $\mathrm{x} 2=0.2$ to keep the $\mathrm{N}$-factor below the critical value of 9 .

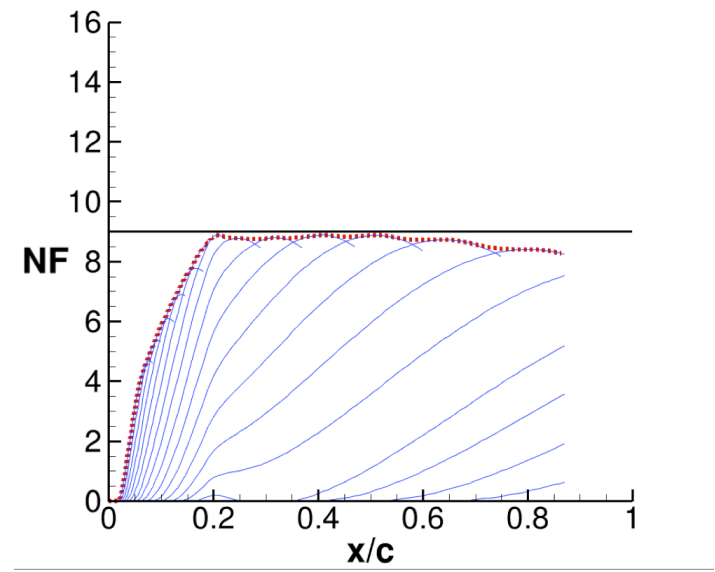

a) TS.

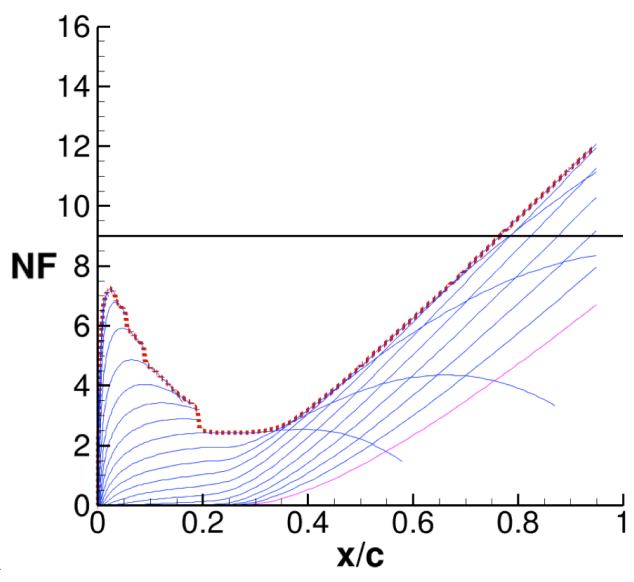

b) $\mathrm{CF}$.

Figure 7 - UDF applied to keep N-factor below 9.

While this is an interesting result and, in theory, would provide full-chord laminar flow, it is not very robust, as the slightest disturbance could push the $\mathrm{N}$-factor above the limit and the transition location would jump forward to $\mathrm{x} / \mathrm{c}=0.2$. In addition, the favorable gradient required to achieve the result in Figure $7 \mathrm{a}$ would cause the CF waves to start growing again as well (Figure 7b). A more practical approach is to move $\mathrm{x} 2$ forward to a location slightly behind $\mathrm{x} 1$ and adjust the UDF parameter to reduce the favorable pressure gradient so that the TS growth envelope curve crosses the critical level at the desired transition location (x3).

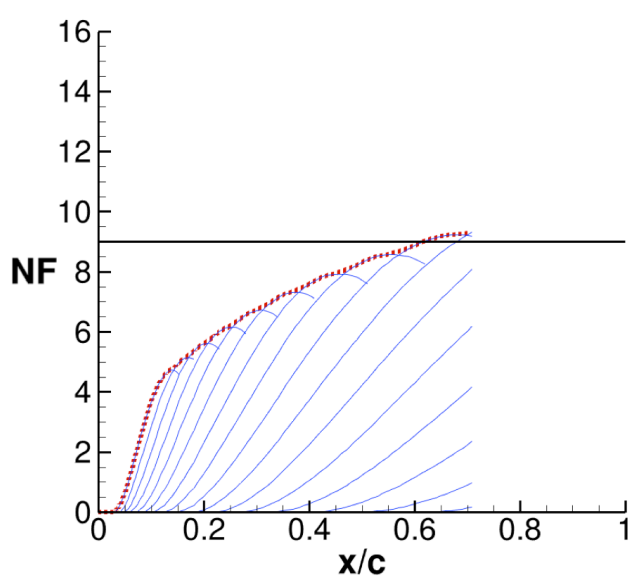

a) TS.

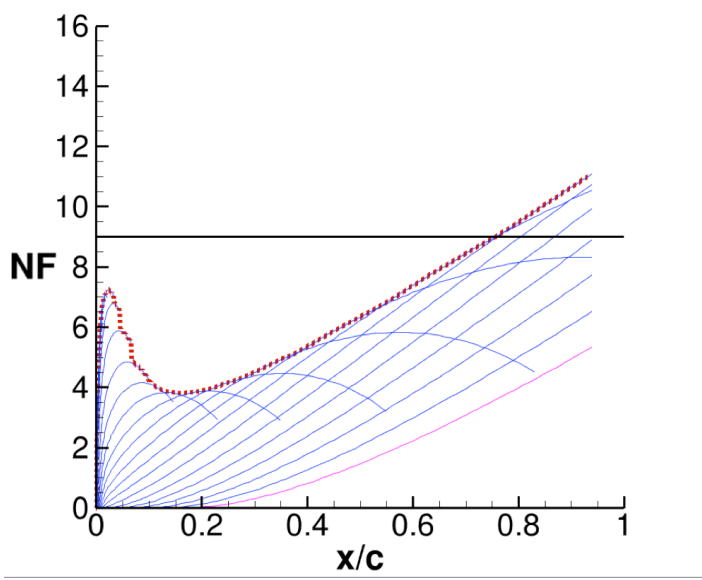

b) $\mathrm{CF}$.

Figure 8 - LASTRAC analysis of alternate target pressure approach.

Results from this alternate approach for a desired transition location of $\mathrm{x} / \mathrm{c}=0.6$ are illustrated in Figure 8. This philosophy has been incorporated into the new CDISC NLF flow constraint and provides a more gradual loss of laminar flow at off-design conditions. Depending on the wing sweep, Reynolds number and critical N-factor level, 
the UDF parameter can actually switch sign to give a positive $\mathrm{C}_{\mathrm{p}}$ increment (adverse gradient) to meet the desired transition location. In addition, the change toward a more positive pressure gradient can help limit increases in wave drag for cases with shocks. An option for automatically limiting the extent of laminar flow to trade viscous drag savings against increases in wave drag is included in the current NLF constraint but was not evaluated in this study.

One additional potential source of boundary layer transition, Görtler vortices, can occur when the flow surface is concave ${ }^{1}$. This is typically not an issue ahead of $\mathrm{x} 3$ on the upper surface of wings, but can be guarded against by imposing a minimum curvature constraint in CDISC.

In the above examples, the baseline $C_{p}$ level was set to zero. For practical transonic wings, the requirements of lift and thickness typically push this level to more negative values corresponding to local Mach numbers that are supersonic. The new NLF constraint in CDISC will automatically adjust the baseline level to match the desired lift, with thickness, curvature and leading-edge radius geometry constraints also affecting the final $\mathrm{C}_{\mathrm{p}}$ levels. Aft of $\mathrm{x} 3$, the NLF constraint blends the target pressure from the value at $x 3$ back to the current wing $C_{p}$ values over the next ten percent chord, with some smoothing employed to reduce kinks at $\mathrm{x} 1, \mathrm{x} 2$ and $\mathrm{x} 3$.

Based on the target pressures in the above example and assuming that issues such as steps, gaps, and surface finish (including insect remains) can be addressed, it appears NLF may be possible at sweep and Reynolds number combinations significantly beyond previously accepted boundaries. In the following section, the achievability and practicality of the method will be evaluated in two transonic transport wing design applications.

\section{Results}

To evaluate the proposed design approach, a wing design case was set up using the Common Research Model (CRM) configuration ${ }^{21}$. This model was originally designed to be representative of current transonic transports and to provide wind tunnel data for use in CFD validation studies. The wing has a leading-edge sweep of 37.3 degrees and the cruise design conditions are a Mach number of 0.85 , a lift coefficient of 0.5 and a Reynolds number of 40 million based on the mean aerodynamic chord. An unstructured viscous grid containing about 11 million tetrahedral cells was generated for the configuration using VGRID for a Reynolds number of 30 million, the highest value reached during the wind tunnel testing program in the National Transonic Facility (NTF) at NASA Langley Research Center. Two designs will be attempted on this configuration at the 30 million Reynolds number, one based on flight turbulence levels and a second based on levels for the NTF wind tunnel.

\section{A. Design at flight conditions}

A fully turbulent analysis was first obtained using USM3D at the design conditions. A series of streamwise design stations were set up at constant intervals out the wing, with an extra station inserted halfway between the last two to give better resolution at the wing tip (see Figure 9). This inserted station is the last one used directly in the design, with changes at the final station and on the tip cap aliased to the inserted station. Similarly, because the wing root is immersed in the fuselage boundary layer, no NLF design is attempted there. However, to guard against attachment line contamination, a leading-edge radius constraint is imposed.

At the active design stations, the new NLF flow constraint is applied with the transition location specified as $\mathrm{x} 3$ $=0.65$ along with a critical $\mathrm{N}$-factor of 13 for both TS and CF. This critical $\mathrm{N}$-factor level is consistent with the value of 14 reported in Belisle ${ }^{6}$ for flight tests with a polished wing leading edge and in Coleman ${ }^{22}$ for results from "quiet" wind tunnels, and probably represents an upper bound on the design space. The NLF constraint also matches a specified lift coefficient at each station, which for this example maintains the original span load. In addition to the NLF flow constraint, geometry constraints were used to maintain the original maximum thickness-to-chord ratio at each station although the chordwise location of the maximum thickness can vary. A leading-edge radius constraint was included to prevent attachment line contamination based on Poll's criteria by keeping the Reynolds number based on boundary layer thickness $\left(\operatorname{Re}_{\theta}\right)$ less than 100 at the two most inboard stations and relaxing the requirement to 235 at the other stations to avoid attachment line transition.

Starting from the initial baseline analysis of 10,000 flow solver iterations, 40 design cycles were run with 400 iterations each, for an additional 16,000 iterations or 1.6 times the original analysis. During the design, the configuration angle of attack was automatically adjusted to maintain the original total lift coefficient. Results from design station 3 on the inboard wing and station 8 on the outboard wing (highlighted in yellow on Figure 9) will be shown in Figures 10-14.

Figure 10a shows the pressure distributions and airfoil shapes at design station 3 for the baseline and final design, along with the target $C_{p}$ distribution. The moderate adverse pressure gradient terminating in a weak shock for the baseline has been replaced by a very mild adverse gradient ending in a slightly stronger shock for the target and design curves. The increase in wave drag due to the stronger shock will reduce the net drag benefit from laminar 
flow at this station. Overall, the target pressures appear to be very well matched by the design result in the NLF region, ahead of $\mathrm{x} 3=0.65$ on the upper surface. Note that $\mathrm{x} 3$ was placed aft of the actual desired transition location of $\mathrm{x} / \mathrm{c}=0.60$ to allow for the effect of smoothing in the target pressure constraint.

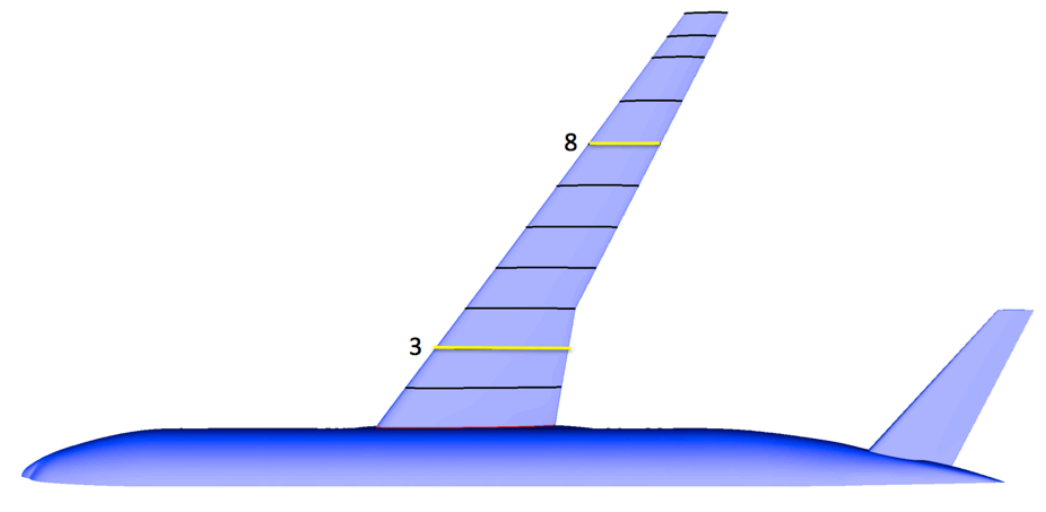

Figure 9 - Wing design station locations.

The baseline and design airfoils are shown in Figure 10b, with the twist having been removed to better illustrate changes in thickness and leading-edge radius. The leading-edge radius on the design airfoil is about half the value for the baseline and there is an undercut on both surfaces until the maximum thickness point, after which the airfoils are very similar. The effects of this smaller leading-edge radius at off-design conditions will need to be evaluated to assess the ultimate practicality of this design.

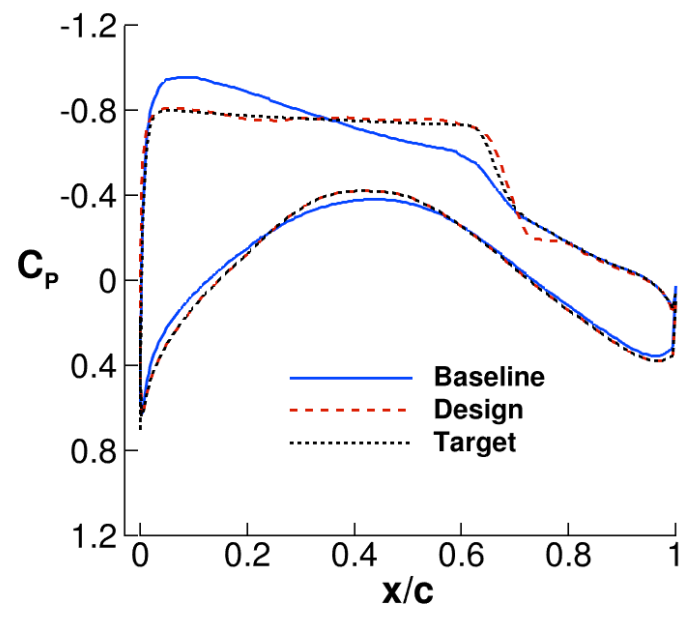

a) Pressure distributions.

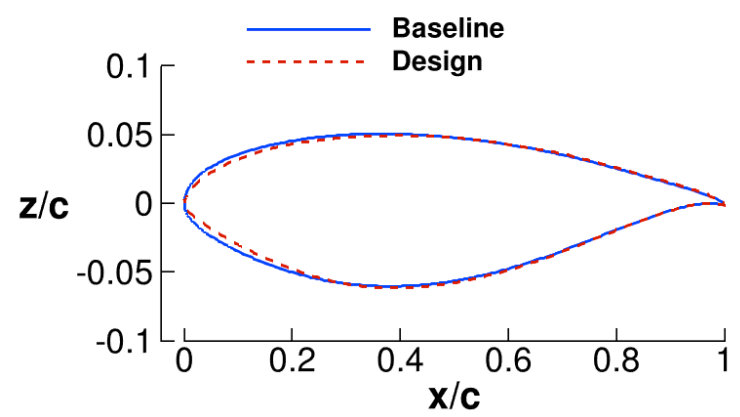

b) Airfoil geometries (twist removed).

Figure 10 - Design results at station 3 (inboard wing).

Plots of TS and CF growth corresponding to the baseline, design and target pressures at station 3 are shown in Figures $11 \mathrm{a}$ and $11 \mathrm{~b}$, respectively. For clarity, only the envelope curves are displayed. For the baseline, the adverse pressure gradient that begins just ahead of $\mathrm{x} / \mathrm{c}=0.1$ (see Figure 10a) leads to rapid growth of the TS curve toward a transition location near $\mathrm{x} / \mathrm{c}=0.25$. The $\mathrm{CF}$ curve grows even more rapidly due to the gradual rounding of the $\mathrm{C}_{\mathrm{p}}$ values ahead of the leading-edge peak seen in Figure 10a, so that, even with the high critical N-factor of 13 , there would be no appreciable laminar flow. The target pressures produce the desired TS growth pattern and transition location while providing a CF margin of 2-3 below the critical limit. The $\mathrm{N}$-factor growth patterns for the final design pressures are fairly similar to the curves for the target pressures. A close inspection of the small mismatches 
in target and design pressures seen in Figure 10a can account for the differences seen in Figure 11 and emphasizes the high level of accuracy required in the design process.

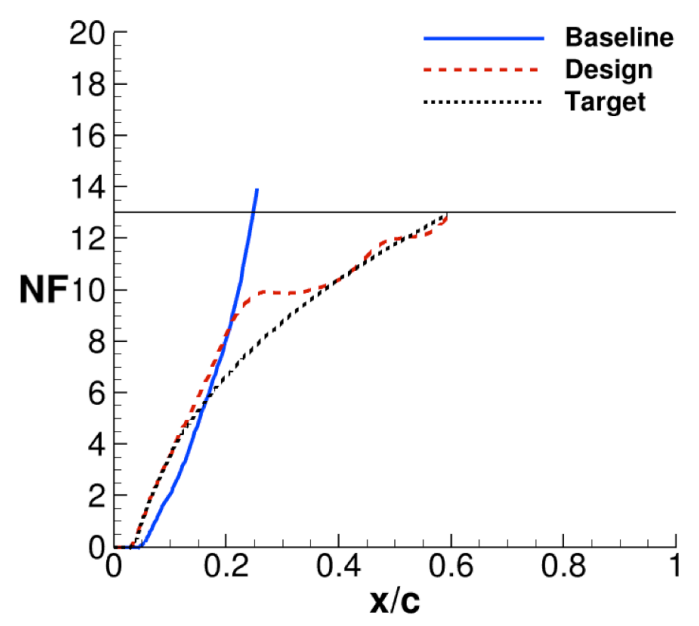

a) TS.

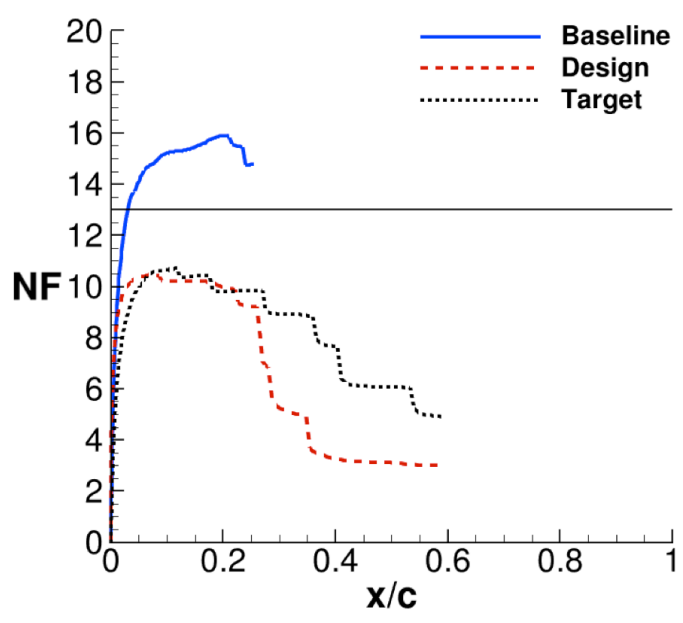

b) $\mathrm{CF}$.

Figure 11 - N-factor growth characteristics at station 3.

At station 8 on the outboard wing panel, the NLF design method generates a target pressure distribution that is very similar to the baseline, but with slightly more adverse gradient (see Figure 12a). As can be seen in Figure 13, the baseline pressure distribution would support laminar flow as both TS and CF levels are below the critical value. The NLF design method could therefore increase the adverse gradient to reduce the wave drag while still meeting the desired transition location. The leading-edge radius had to be reduced slightly (Figure 12b) to meet the attachment line restriction, but the baseline and final design airfoils are otherwise very similar. For TS, the design and target curves in Figure 13a are very similar, but the CF levels for the design case in Figure 13b are noticeably higher than the target values. The reason for this discrepancy was not initially obvious as the target and design pressures appear to be closely matched. This issue will be addressed in the section on the design for wind tunnel conditions.

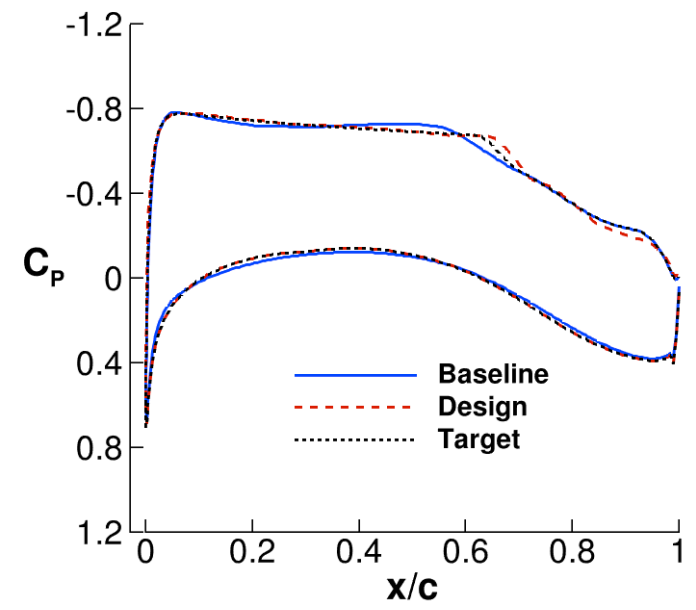

a) Pressure distributions.

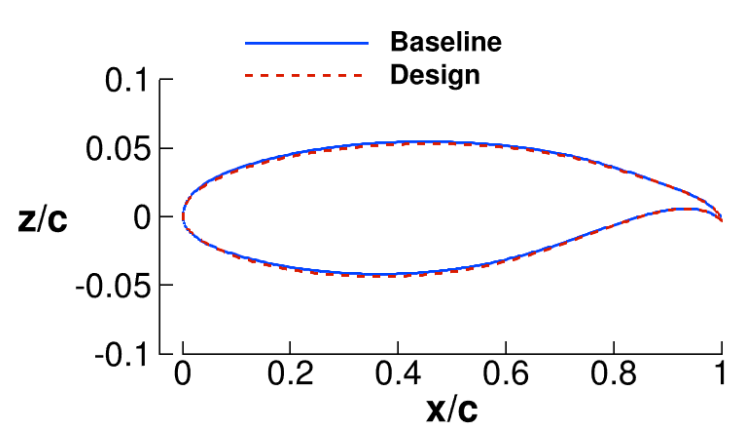

b) Airfoil geometries (twist removed).

Figure 12 - Design results at station 8 (outboard wing). 


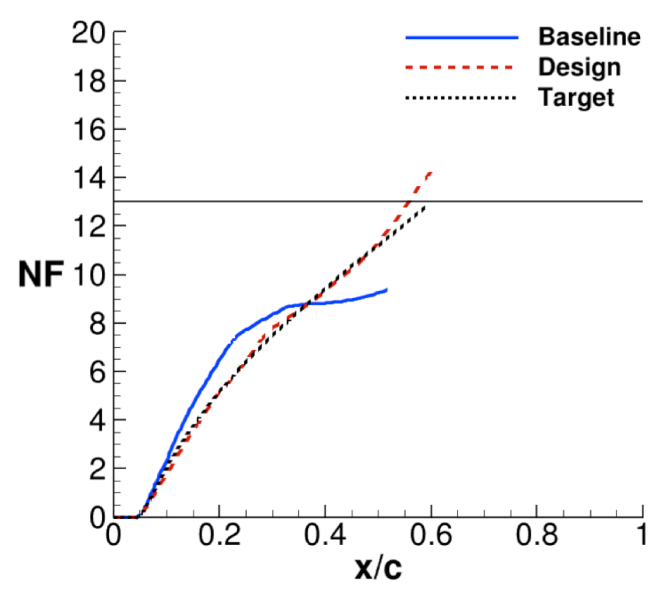

a) TS.

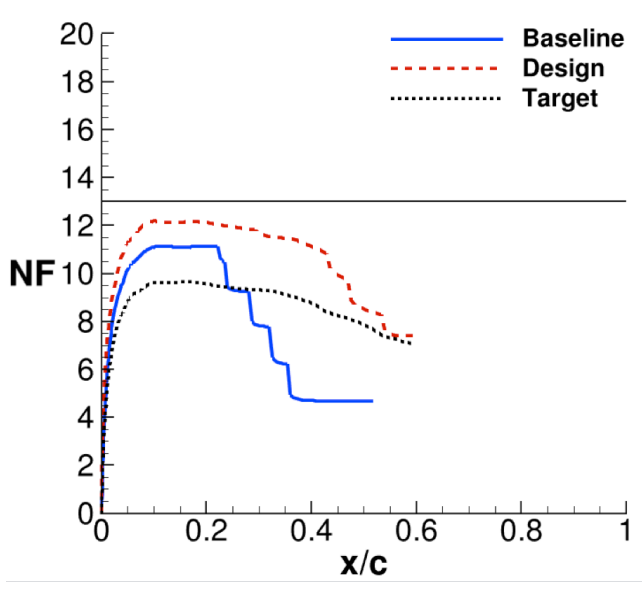

b) CF.

Figure 13 - N-factor growth characteristics at station 8 (outboard wing).

For this initial exercise of the transonic NLF design process, the design started from the fully turbulent baseline solution but with a new transition front defined on the upper surface of the wing based on the LASTRAC analysis for the target pressure distributions. The final design matched the original baseline lift coefficient of 0.5 within 0.001 . The drag coefficient for the design was 0.0217 compared to 0.0238 for the baseline, or a drag reduction of 21 counts. Turbulent analysis of the final design, representative of the loss of all laminar flow on the wing, gave a drag coefficient of 0.0244 , or a 6 -count penalty. The turbulent run required about 0.37 degrees more angle of attack relative to the laminar run to match the desired lift coefficient. Some preliminary analyses indicate that the above drag penalty could be cut in half by deflecting aft control surfaces, but a systematic study is needed for a better assessment.

Pressure distributions at stations 3 and 8 for the laminar and turbulent flow analyses of the final design are shown in Figure 14. At station 3, the pressure distributions are fairly similar, with the turbulent case shock slightly stronger and further forward. At station 8, the turbulent shock is even further forward and stronger as the $\mathrm{C}_{\mathrm{p}}$ rooftop level required more suction to maintain lift. At both stations, there is a small expansion on the upper surface just ahead of the trailing edge for the laminar case, which is consistent with a thinner turbulent boundary layer there. The thinner boundary layer at the trailing edge will reduce the profile drag, adding to the overall drag savings.

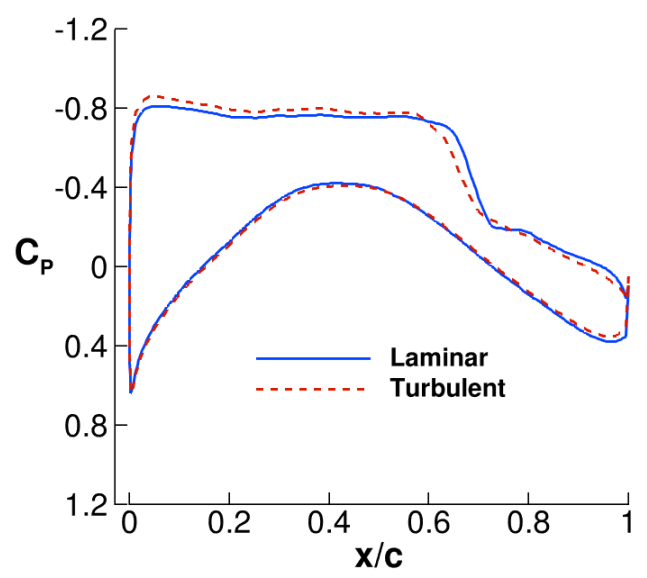

a) Station 3 .

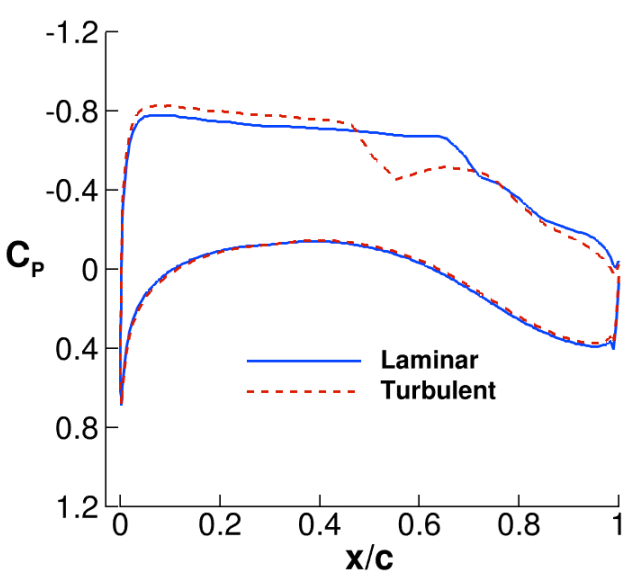

b) Station 8 .

Figure 14 - Comparisons of laminar and turbulent pressure distributions for the final design geometry. 
Overall, the design method was successful at matching the target pressures with sufficient accuracy to achieve the desired amount of laminar flow. Figure 15 shows a plot of the transition fronts on the wing planform corresponding to the target and final design pressure distributions. Other than at the first station outboard of the root, where a small oscillation in pressure caused an early termination of the boundary layer solution, the target transition locations are closely matched. Thus, the first two questions posed at the outset of this study concerning the ability to both create and match NLF target pressure distributions appear to be answered affirmatively.

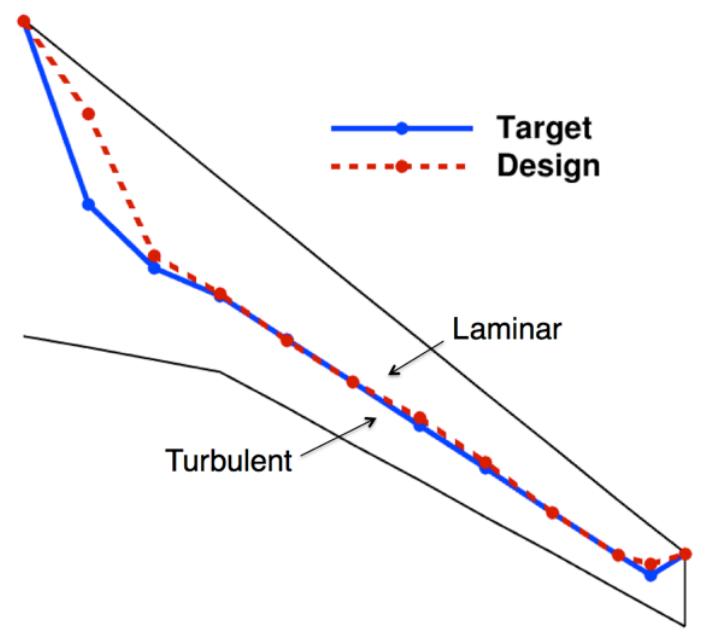

Figure 15 - Comparison of the transition fronts for target and design pressures.

The third question concerning attachment line contamination and transition is addressed in Figure 16, where the spanwise variation of $\mathrm{Re}_{\Theta}$ for the attachment line for both the baseline and design is plotted. All of the values for both configurations are below the 235 transition limit (solid black line) except at the wing root, implying that if a device such as a Gaster bump was used inboard, most of the wing would remain laminar. It is interesting to note that a leading-edge radius constraint was applied to the root station in the design, but no actual design was done there and the attachment line remained on the lower surface in a lower curvature area resulting in a higher value of $\mathrm{Re}_{\Theta}$. This emphasizes the need to have the attachment line very near to the leading edge for the radius constraint to be effective in controlling the attachment line state. The rest of the design wing has $\operatorname{Re}_{\Theta}$ values close to the contamination limit of 100 (dashed black line), below which the flow will convert to laminar even if previously tripped. Perhaps some further design could bring all of these values below 100 and eliminate the need for a Gaster bump. Some additional options for design near the attachment line are discussed in the next section.

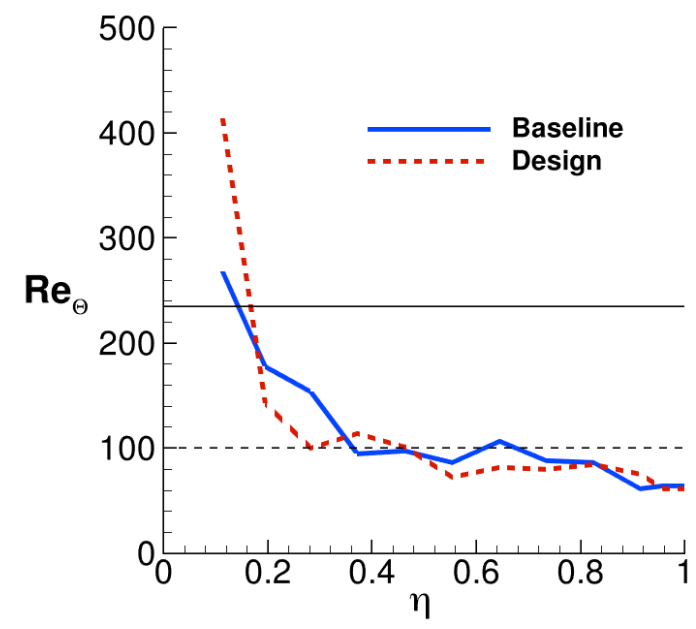

Figure 16 - Spanwise variation of attachment line $R_{\Theta}$ values. 
In Figure 17, the transition Reynolds numbers corresponding to the target pressures across the wing for this design have been added to Figure 1 at the CRM wing sweep value of 37.3 degrees. The value near the root of the wing (top of the red line) is about a 5-fold increase relative to the current boundary at the same sweep or, alternatively, it requires the sweep to be reduced to about 10 degrees to obtain this $\mathrm{Re}_{\mathrm{T}}$ level. While there are still questions to be answered concerning the viability of this approach, and of laminar flow in general, the significant drag reduction obtained using NLF at this wing sweep seems to indicate that further evaluation of the method is warranted.

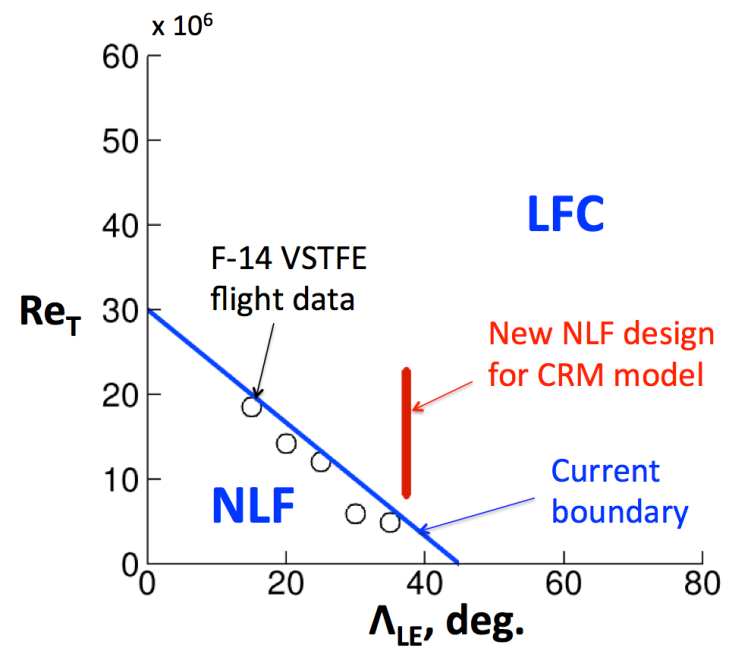

Figure 17 - New design relative to current NLF-LFC boundary.

\section{B. Design for wind tunnel testing in the NTF}

In light of the encouraging results from the above study, interest has been expressed in verifying the design method experimentally. Previous NLF testing in the NTF gave a preliminary estimation of critical N-factors for TS and $\mathrm{CF}$ in the range of 6 , less than half of the values used in the above design study as representative of flight. It is anticipated that a new NLF CRM model, if built, would be a semispan model to allow higher chord Reynolds numbers for a given tunnel unit Reynolds number to help alleviate turbulent wedges seen in the previous test, which should allow a more accurate determination of these values for the NTF. The following is a discussion of the work to date on developing an outer mold line for the proposed model.

The CDISC NLF constraint includes the effects of critical N-factors in determining the extent of the initial acceleration (x1) for CF control and the UDF values for limiting TS growth. The algorithms for determining these values were calibrated on a supersonic configuration for $\mathrm{N}$-factors of 9-13 and did not produce the desired transition front in the initial design attempt. After a few manual adjustments to these parameters, it was found that the TS growth could be controlled to meet the desired transition location at a critical N-factor of 6 , but that the CF $\mathrm{N}$-factor peak near the leading edge was still too high at every station on the wing (see Figure 18 for examples). 


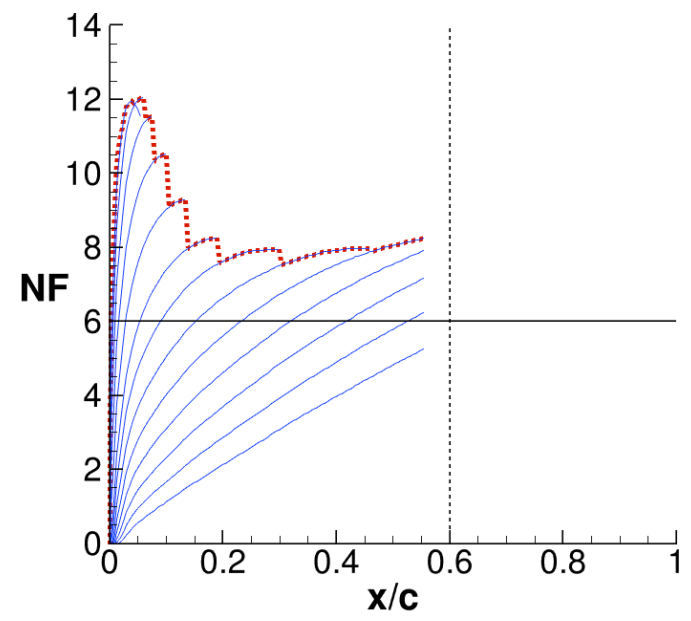

a) Station 3 .

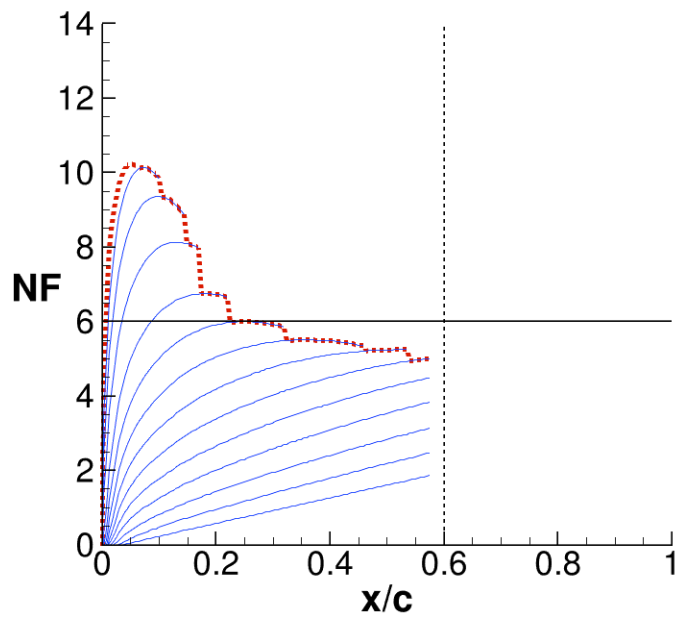

b) Station 8 .

Figure 18 - CF N-factor growth characteristics for initial design for wind tunnel model.

In order to investigate this problem in a timely manner, it was decided to run USM3D using a quasi-2D conical flow (2.75D) approach similar to that of Streit ${ }^{23}$. For this approach, the Q2D grid generation method ${ }^{24}$ was used to create a one-cell wide grid for an airfoil section from the wing with the points on the second "symmetry" plane sheared and compressed to match the 3D wing leading- and trailing-edge sweeps. The periodic boundary condition available in USM3D was used on the vertical planes in place of the normal symmetry plane conditions. As noted by Streit, this provides the correct leading-edge sweep that is important for CF as well as a good approximation to the mid-chord shock sweep, assuming that the 3D wing flow isobars approximate lines of constant $\mathrm{x} / \mathrm{c}$. Because this gridding approach only requires one cell in the spanwise direction, the grid spacing around the leading edge of the wing in the vertical planes was made considerably finer than that used in the 3D cases, providing greater resolution of the flow near the attachment line and in the initial upper surface acceleration region while still maintaining a reasonable turn-around time. This approach is most applicable to the outboard panel of the wing, away from the fuselage, planform break and wing tip that would tend to make the flow less conical in character.

A review of the results on the outboard wing stations from the initial 3D design at the tunnel critical $\mathrm{N}$-factor of 6 indicated that design station 6 had one of the higher CF peaks, so the airfoil at this station was selected for the study. The chord Reynolds number at this station is 25.5 million and the leading- and trailing-edge sweeps are 37.3 and 27.3 degrees, respectively. The angle of attack for the $2.75 \mathrm{D}$ case was adjusted to approximate the $3 \mathrm{D} \mathrm{C}_{\mathrm{P}}$ levels for this station and then a design was performed to bring the pressure distribution into close agreement with the CDISC target pressures. The resulting pressure distributions are shown in Figure 19. Although it appears that the final design pressures are in good agreement with the target in general (see Figure 19a), a close-up view of the pressure distributions near the leading edge (Figure 19b) indicates that there are discrepancies between the target pressures (black short-dashed line) and design pressures (red dashed line). One such discrepancy is the $\mathrm{x} / \mathrm{c}$ location of the maximum $\mathrm{C}_{\mathrm{P}}$, corresponding to the attachment point. For the target pressures, the attachment point is prescribed to be at the leading edge (point B), but for the design pressures, the attachment point occurs aft of the leading edge on the lower surface (point A). A second discrepancy between the target and design pressures can be seen on the upper surface between points $\mathrm{C}$ and $\mathrm{D}$. The design pressures are significantly more positive than the target pressures between where the target and design pressure distributions first cross (point C) and where they are both relatively flat (point $\mathrm{D}$ ). This illustrates that the design pressures did not successfully match the rapid acceleration very near the leading edge that was prescribed by the target pressures. The corresponding stability analysis results shown in Figure 20a reflect the observations from the initial 3D design that the TS growth curves are suppressed below the critical $\mathrm{N}$-factor level of 6 until the desired transition location (vertical dashed line at $\mathrm{x} / \mathrm{c}=$ 0.6). However, Figure $20 \mathrm{~b}$ shows that the $\mathrm{CF}$ curves grow beyond the critical $\mathrm{N}$-factor very close to the leading edge.

In order to investigate the impact of these discrepancies between the target and design pressures, three additional stability analyses were performed with the CF results plotted in Figure 21. For the following discussion, refer to 
Figure $19 \mathrm{~b}$ for the definition of the various segments of the pressure distributions. In the first case, the target pressures from B-C were substituted for the design pressures from A-C to assess the effect of the attachment line being offset from the leading edge. The second case kept the design pressures from A-C, but substituted the target pressures (black short-dashed line) for the design pressures (red dashed line) from C-D to assess the mismatch on the upper surface. The final case essentially combines the first two cases using the target pressures from B-C-D. As can be seen, the use of the target pressures significantly reduces the resulting CF peaks, with the B-C-D substitution case bringing down the peak by a factor of 5 relative to the original design.

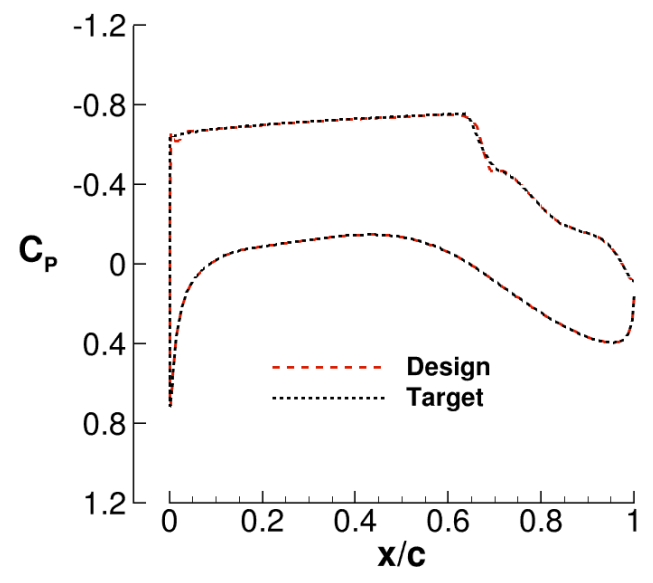

a) Full chord.

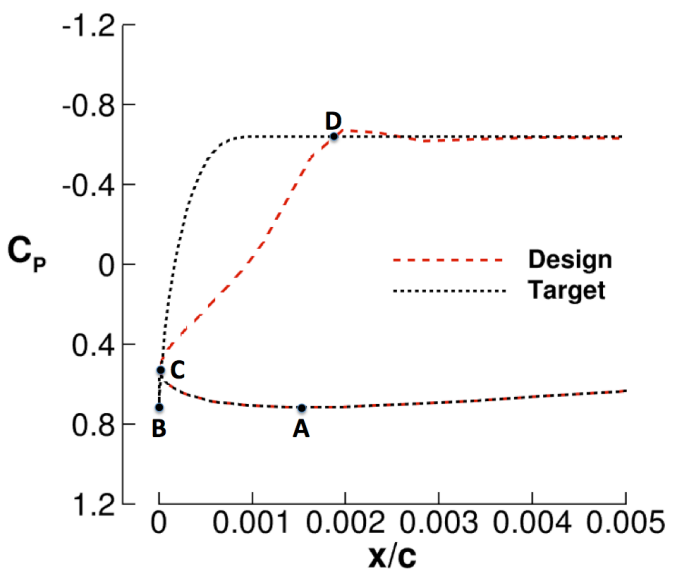

b) Leading edge.

Figure 19 - Pressure distributions for 2.75D design of station 6 airfoil.

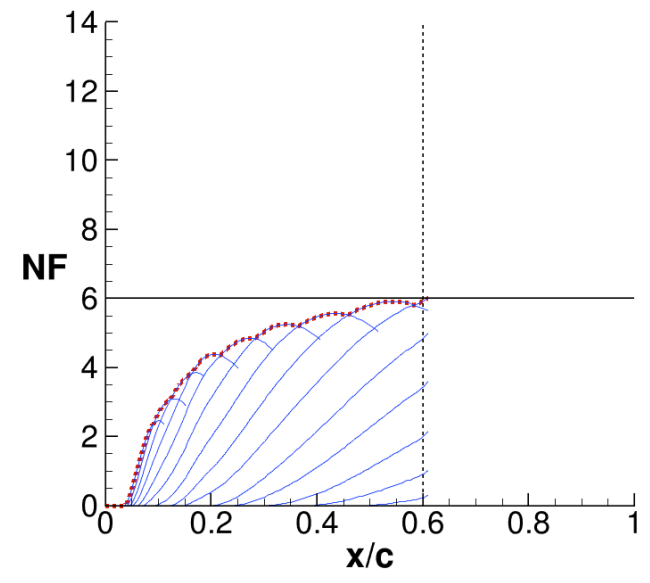

a) TS.

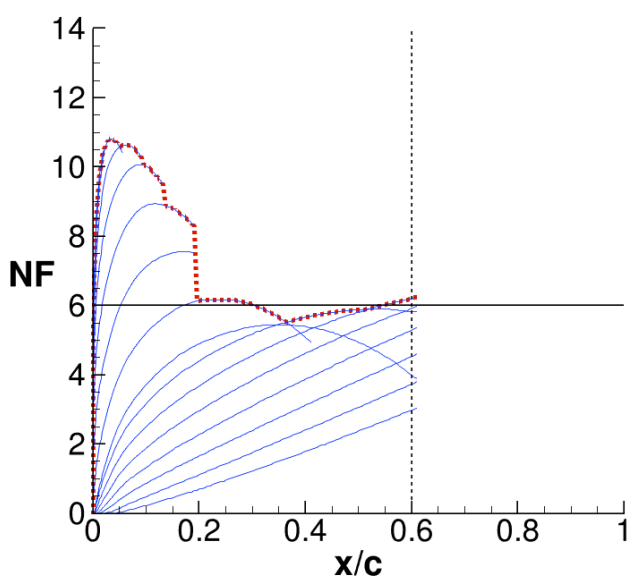

b) $\mathrm{CF}$.

Figure 20 - Stability analysis results for 2.75D design of station 6 airfoil. 


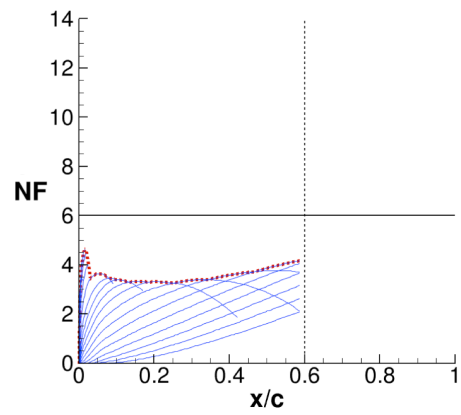

a) B-C only.

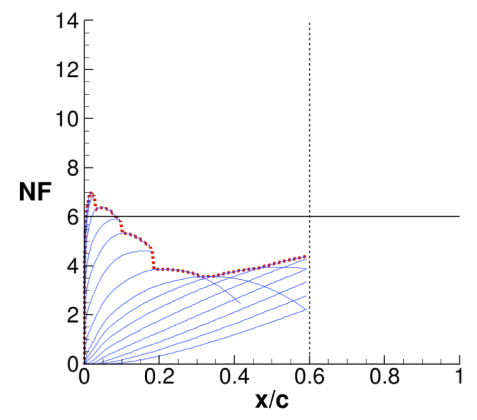

b) C-D only.

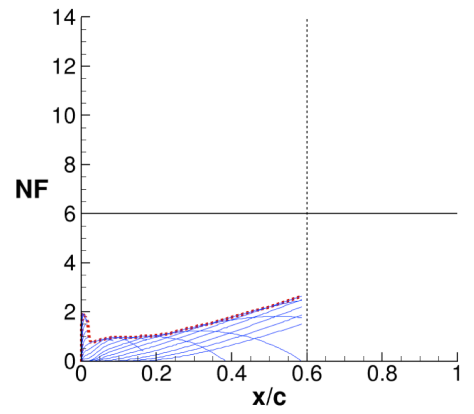

c) B-C-D.

Figure 21 - Stability analysis results for modified pressure distributions.

As the original CDISC design algorithm was not able to match the NLF target pressures with sufficient accuracy, an additional constraint was developed to modify the geometry very near the leading edge $(\mathrm{x} / \mathrm{c}<0.005)$ on the upper and/or lower surface in order to obtain better agreement with the target pressures. A bump was added to each surface (upper and lower) with the shape based on differences between the target and design pressure coefficients, with the lower surface option also deflecting both the leading edge and nearby upper surface points downward to try to drive the attachment point to the leading edge.

Three additional 2.75D designs were performed using the original constraints along with the new constraint for the top bump only, the bottom bump only and both top and bottom bumps (2-bump case). The results are shown in Figures 22-24, with baseline referring to the design with the original constraints (no bumps). Note that the z/c scale in the geometry plots has been compressed relative to $\mathrm{x} / \mathrm{c}$ to better illustrate the bumps. In all three cases, the bumps provide an improved match to the target pressures in their areas of influence relative to the original design (i.e., the top bump provided a better match to the upper surface target segment (black short-dashed line) in Figure 19b, while the bottom bump improved the match near the target attachment point B, also in Figure 19b).

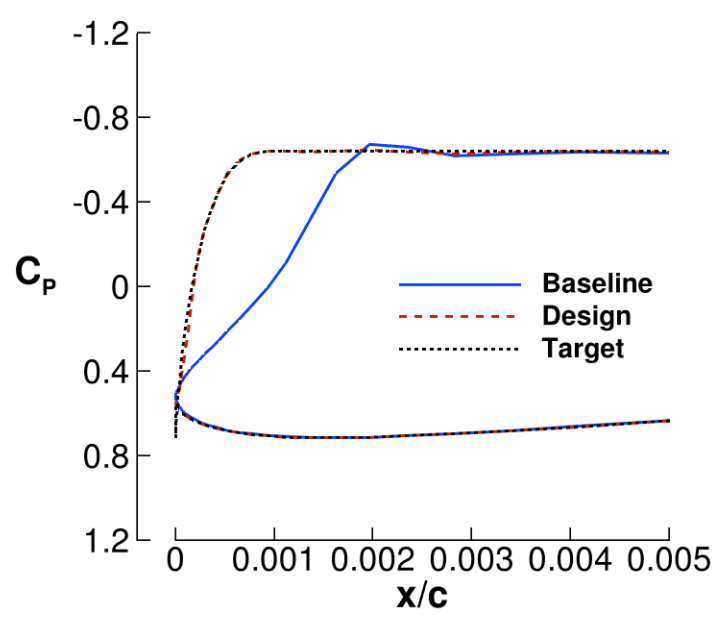

a) Pressures.

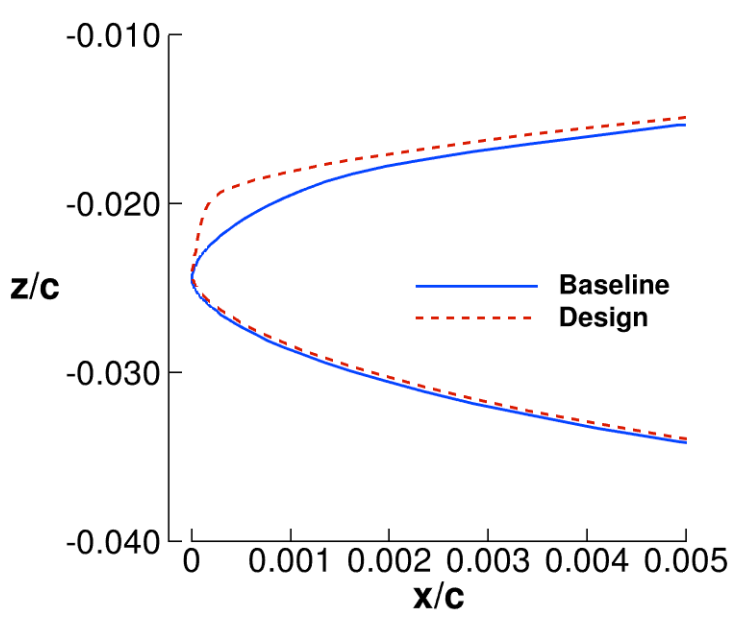

b) Geometry.

Figure 22 - Design results for the top bump case. 


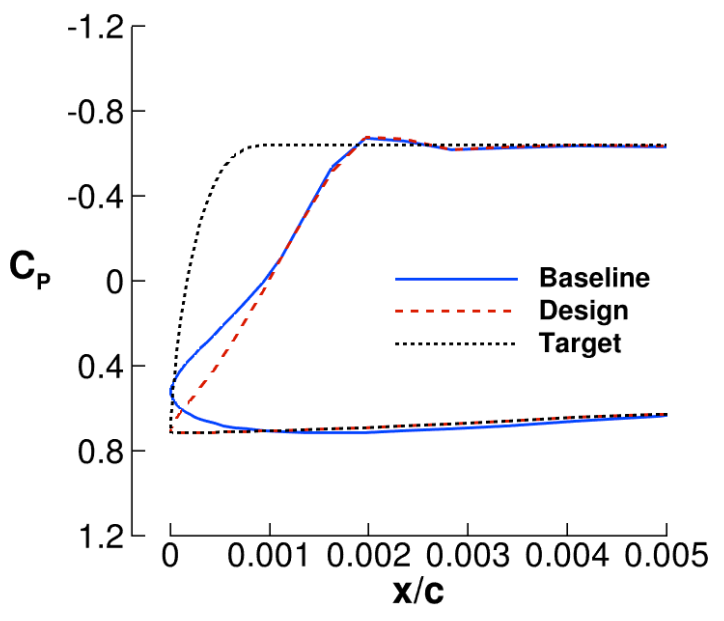

a) Pressures.

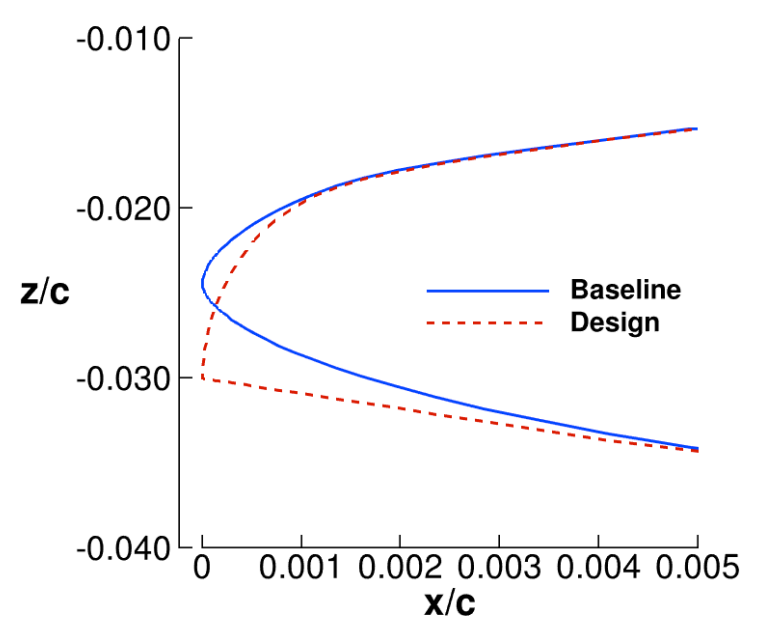

b) Geometry.

Figure 23 - Design results for the bottom bump case.

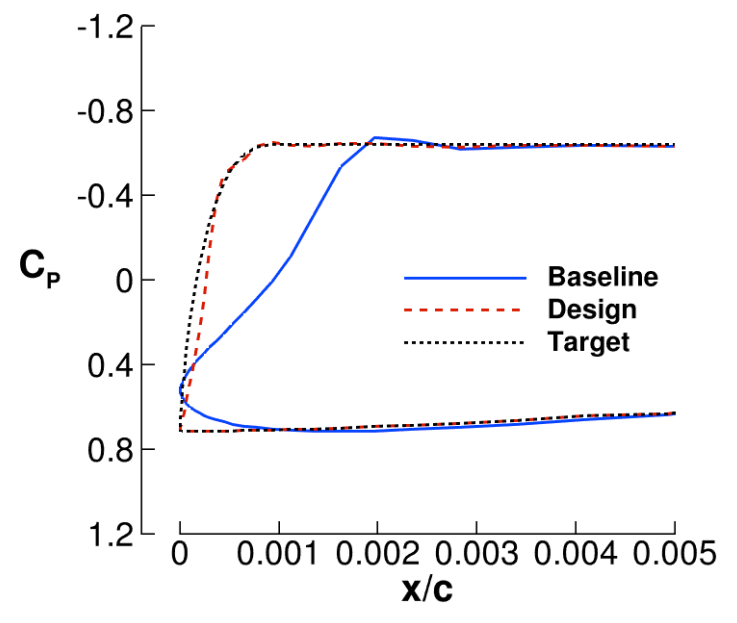

a) Pressures.

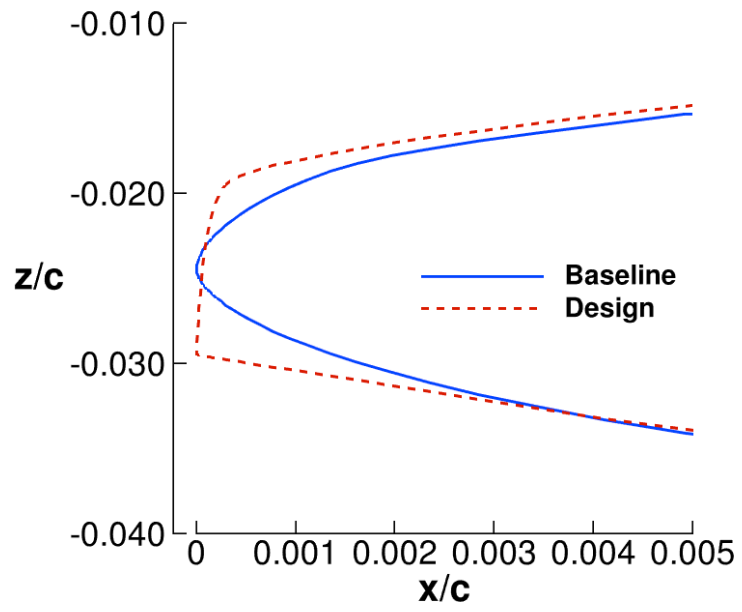

b) Geometry.

Figure 24 - Design results for the 2-bump case.

As a result of the improved matches to the target pressures, the CF peaks at the leading edge have also been reduced (not shown), though not to the degree that would have been suggested by the target pressure substitution study results shown in Figure 21. The reason for this shortfall is examined using Figure 25 below. Recall from Figure 4 that the algorithm for determining CF peak height was based on the $\mathrm{x} 1$ parameter, which is arc length from the attachment point to the level-pressure region. Figure 25 a plots the standard $C_{P}$ versus chord fraction $(x / c)$ for the no bump case (blue line) and two versions of the 2-bump case. Figure $25 \mathrm{~b}$ plots the same pressures against arc length $(\mathrm{s} / \mathrm{c})$, which is consistent with the $\mathrm{x} 1$ parameter used to predict CF peak height in Figure 4. The case labeled "2-bump target" (red dashed line) is for the 2-bump target pressures applied to the starting geometry (no bump) and represents the change that was originally expected from the design if the target pressures could be met. The case labeled "2-bump design" shown by the black short-dashed line is the final 2-bump design result. As can be seen in the figure, even though the target pressures were closely matched by the design in terms of $\mathrm{x} / \mathrm{c}$ (Figure 25a), there is a considerable spread between them in terms of $\mathrm{s} / \mathrm{c}$ (Figure 25b). From Figure 25b, the $\mathrm{x} 1$ value for the 2-bump design is 0.010 instead of the value for the target of 0.005 , giving only about one-third of the anticipated change from the original no-bump $\mathrm{x} 1$ value of 0.012 . 


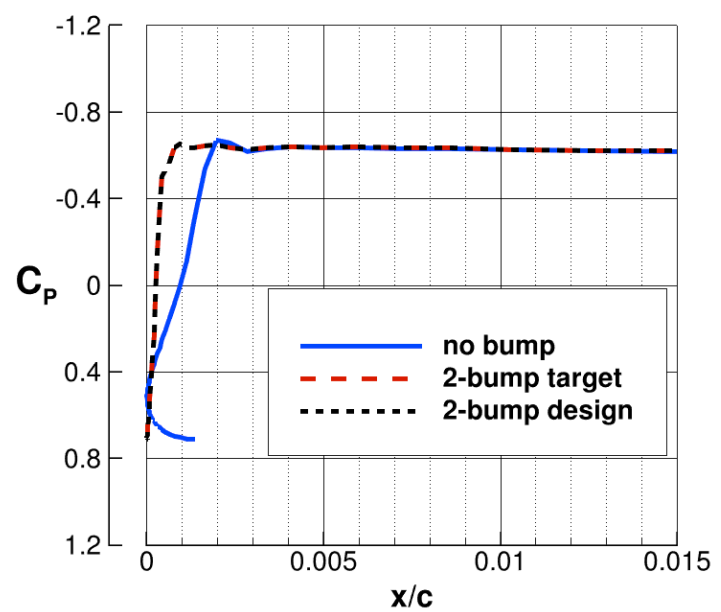

a) Pressure distributions using chordwise fraction.

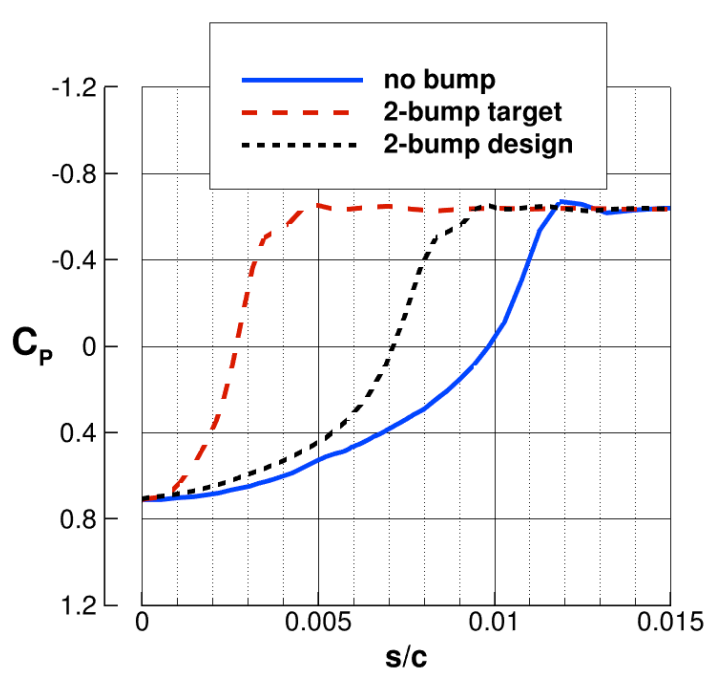

b) Pressure distribution using arc length.

Figure 25 - Effects of airfoil geometry on pressure distribution.

This variation is due to the thickening of the airfoil at the leading edge during the design as the bumps are added to match the target pressures, thus creating a longer arc length from the attachment point to the $\mathrm{x} 1$ location where the pressures level off. This issue did not occur in earlier designs because the leading-edge radius constraint was active, maintaining a near-constant thickness near the leading edge throughout the design. The impact of this difference on the CF leading-edge peak values can be seen in Figure 26, where the envelope curves for the three cases are plotted. Consistent with the variation of $\mathrm{x} 1$ seen in Figure 25b, the drop in CF peak height from 11 for the no bump case to 8 for the design case is only about one-third of the expected change to the value of 2 predicted for the target pressures at the beginning of the design.

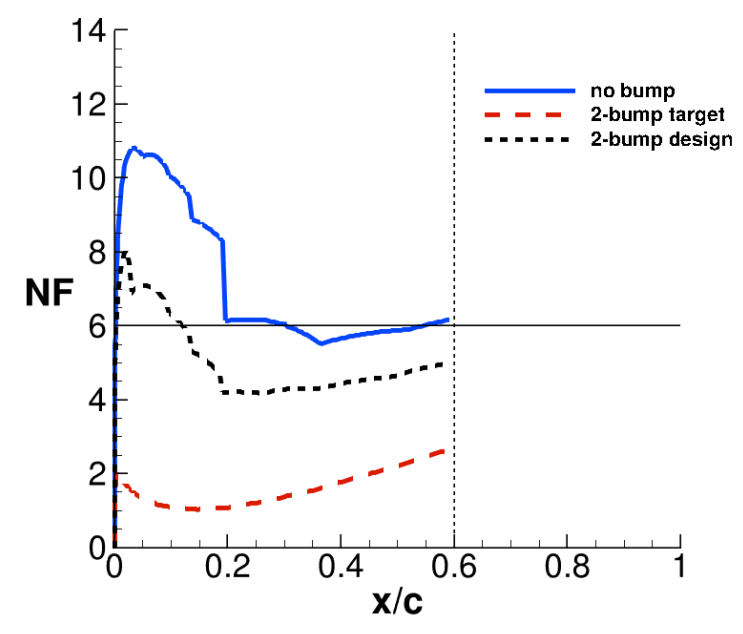

Figure 26 - Effects of airfoil geometry on CF peak height.

As the 2-bump airfoil already has a fairly blunt leading edge, it is questionable if a further reduction in $\mathrm{x} 1$ is practical, as continued reductions in $\mathrm{x} / \mathrm{c}$ in the target pressures seem to be offset to a large degree by increases in leading-edge thickness, with corresponding increases in arc length. While this remains a topic of investigation, it should be noted that the design approach can still be evaluated experimentally by reducing the test Reynolds number. Figure 27 indicates that a linear scaling of the Reynolds number by the ratio of the current and desired Nfactor peaks, lowering it from 25.5 to 17 million, produces the desired $\mathrm{N}$-factor growth curve results. 


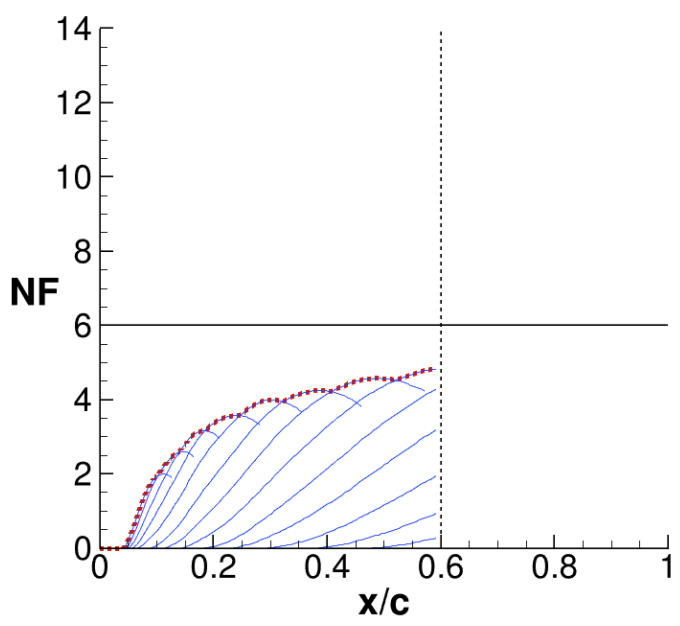

a) TS.

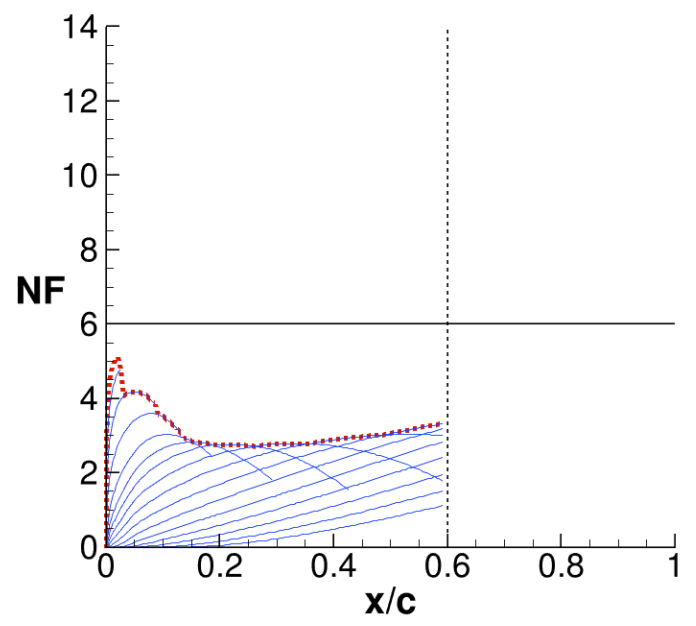

b) $\mathrm{CF}$.

Figure 27 - Stability analysis of 2-bump design at a Reynolds number of 17 million.

While the above results indicate that the current design approach can do a reasonable job of matching NLF target pressures, they do emphasize that the actual geometry is a key component of the definition. This is true as well in addressing the question of attachment line boundary layer state. The original imposition of a leading-edge radius constraint based on Poll's criteria is really only valid if the attachment line is at the leading edge. In the above study, this is only true for the cases using the lower surface bump (bottom bump and 2-bump). The values of $\operatorname{Re}_{\Theta}$ at the attachment line were extracted from the BLSTA3D results for each of the cases (no bump, top bump, bottom bump, 2-bump). All of the values are below the 235 limit used for the outboard airfoils, but only the bottom bump and 2bump cases fall below the 100 level that protects against contamination and would be needed inboard. While a design with a bottom bump potentially could alleviate the need for a Gaster bump or similar device, the unique shape of all of the bump airfoils strongly suggests that further evaluation at other conditions is needed to assess their robustness. Application of the lessons learned from these 2.75D studies will be reflected in the $3 \mathrm{D}$ design as it is continued.

\section{Concluding Remarks}

This study was initiated to assess the feasibility of applying an NLF design method for supersonic vehicles to a configuration representative of current commercial transonic transports. The following is a summary of where we are in addressing the three key questions posed in Section II. NLF Design Approach.

In answer to the first question, it appears that, in general, the new method does a good job of defining target pressures that can meet the NLF goals as well as other flow constraints. There was a slight wave drag penalty incurred in the design at flight conditions as expected, but the NLF benefit easily outweighed it. The design for the wind tunnel model at the lower critical $\mathrm{N}$-factor level did indicate that the target pressure in terms of $\mathrm{x} / \mathrm{c}$ cannot be fully defined a priori and that there may be a limit in the CF peak reduction at some conditions.

Relative to the second question, it also appears that the original CDISC design method could match the target pressures with sufficient accuracy at flight conditions to achieve the desired extent of laminar flow. At the wind tunnel conditions, which required more aggressive pressure gradients near the leading edge, a new leading-edge bump constraint acted as an auxiliary design driver to provide a very accurate match to the target pressures in this area also. However, the unique leading-edge geometries produced did not always provide the anticipated NLF benefits. The typical geometry constraints such as maximum airfoil thickness, twist and leading-edge radius were easily met in all cases, although for the bump cases the airfoil leading-edge geometry may call into question existing correlations of aerodynamic characteristics with leading-edge radius.

For the third question, it appears that meeting the attachment line transition constraint $\left(\operatorname{Re}_{\Theta}<235\right)$ is not a major problem, with many of the baseline CRM airfoil sections already close to or below the 100 level required to prevent attachment line contamination. It did become obvious, however, that it is not just the leading-edge radius value that 
is important, but also the location of the attachment line relative to the leading edge and the velocity gradients at that point. The new bump constraint seems to offer some potential for better control of the attachment line $\mathrm{Re}_{\Theta}$, perhaps eliminating the need for a Gastor bump if the higher values of $\mathrm{Re}_{\Theta}$ occurring at the wing root could be reduced below 100 .

Finally, while the above results are encouraging with respect to the NLF design method performance, further assessment of any design is needed relative to robustness at off-design conditions and manufacturing and maintenance of the resulting wing. The design results indicate that small changes in pressure can have a significant impact on the resulting $\mathrm{N}$-factor curves, so tight control on contour tolerances as well as attention to surface finish will be needed.

\section{Acknowledgments}

This research was conducted as part of the High Aspect Ratio Wing Subproject work within the NASA Advanced Air Transportation Technology (AATT) Project. The authors would also like to thank Dr. Meelan M. Choudhari and Dr. Chau-Lyan Chang for their invaluable help in running and understanding the results from the LASTRAC code.

\section{References}

${ }^{1}$ Joslin, Ronald D.: Oveview of Laminar Flow Control. NASA TP-1998-208705, October 1998.

${ }^{2}$ Crouch, J.D.: Boundary-Layer Transition Prediction for Laminar Flow Control. AIAA 2015-2472, June 2015.

${ }^{3}$ Collier quote from Green Aviation TIM - waiting for word from Fay if it is available to public

${ }^{4}$ Seitz, Arne; Kruse, Martin; Wunderlich, Tobias; Bold, Jens; and Heinrich, Lars: The DLR Project LamAiR: Design of a NLF Forward Swept Wing for Short and Medium Range Transport Application. AIAA 2011-3526, June 2011.

${ }^{5}$ Saric, William S.; Carrillo, Ruben, B., Jr.; and Reibert, Mark S.: Leading-Edge Roughness as a Transition Control Mechanism. AIAA 1998-0781, January 1998.

${ }^{6}$ Belisle, Michael J.; Roberts, Matthew W.; Williams, Thomas C.; Tufts, Matthew W.; Tucker, Aaron A.; Saric, William S.; and Reed, Helen L.: A Transonic Laminar-Flow Wing Glove Flight Experiment: Overview and Design Optimization. AIAA 2012-2667, June 2012.

${ }^{7}$ Anderson, Bianca Trujillo; and Meyer, Robert R.: Effects of Wing Sweep on In-Flight Boundary-Layer Transition for a Laminar Flow Wing at Mach Numbers from 0.60 to 0.79. NASA Technical Memorandum 101701, July 1990.

${ }^{8}$ Campbell, Richard L.; Campbell, Matthew L.; and Streit, Thomas: Progress Toward Efficient Laminar Flow Analysis and Design. AIAA 2011-3527, June 2011.

${ }^{9}$ Vermeersch, Olivier; Yoshida, Kenji; Ueda, Yoshine; and Arnal, Daniel: "Natural laminar flow wing for supersonic conditions: Wind tunnel experiments, flight test and stability computations". Progress in Aerospace Sciences (2015), http://dx.doi.org/10.1016/j.paerosci.2015.07.003i.

${ }^{10}$ Ueda, Yoshine; Yoshida, Kenji; Matsushima, Kisa; and Ishikawa, Hiroaki: "Supersonic Natural-Laminar-Flow WingDesign Concept at High-Reynolds-Number Conditions". AIAA Journal, Vol. 52, No. 6, pp. 1294-1306, June 2014.

${ }^{11}$ Campbell, Richard L., "Efficient Viscous Design of Realistic Aircraft Configurations," AIAA-98-2539, June 1998.

${ }^{12}$ Frink, N.T., Pirzadeh, S.Z., Parikh, P.C., Pandya, M.J., and Bhat, M.K., "The NASA Tetrahedral Unstructured Software System," The Aeronautical Journal, Vol. 104, No. 1040, October 2000, pp.491-499.

${ }^{13}$ Pirzadeh, S., "Three-Dimensional Unstructured Viscous Grids by the Advancing-Layers Method," AIAA Journal, Vol. 34, No. 1, January 1996, pp.43-49

${ }^{14}$ Frink, N.T., "Assessment of an Unstructured-Grid Method for Predicting 3-D Turbulent Viscous Flows," AIAA-96-0292, January 1996.

${ }^{15}$ Spalart, P., and Allmaras, S.A., "One-equation turbulence model for aerodynamic flows," AIAA 92-0439, January 1992.

${ }^{16}$ Pandya, Mohagna J.; Abdol-Hamid, Khaled S.; Campbell, Richard L.; and Frink, Neal T.: Implementation of Flow Tripping Capability in the USM3D Unstructured Flow Solver. AIAA-2006-0919, January 2006.

${ }^{17}$ Chang, C.-L., "The Langley Stability and Transition Analysis Code (LASTRAC): LST, Linear and Nonlinear PSE for 2-D, Axisymmetric, and Infinite Swept Wing Boundary Layers," AIAA Paper 2003-0974, 2003.

${ }^{18}$ Wie, Y.-S., "BLSTA: A Boundary Layer Code for Stability Analysis," NASA CR 4481, 1992.

${ }^{19}$ Poll, D. I. A. : Some Observations of the Transition Process on the Windward Face of a Long Yawed Cylinder. J. Fluid Mech., vol. 150, pp. 329-356, 1985.

${ }^{20}$ Gaster, M.: On the Flow Along Swept Leading Edges. Aeronaut. Q., vol. XVIII, pt. 2, pp. 165-184, 1967.

${ }^{21}$ Vassberg, John C.; and Rivers, S. Melissa: Development of a Common Research Model for Applied CFD Validation. AIAA 2008-6919, August 2008.

${ }^{22}$ Coleman, Colin P.: Boundary Layer Transition in the Leading Edge Region of a Swept Cylinder in High Speed Flow. NASA TM-1998-112224, March 1998.

${ }^{23}$ Streit, Thomas; Wichmann, Georg; zu Hatzbach, Fedime von Knoblauch; and Campbell, Richard L.: Implications of Conical Flow for Laminar Wing Design and Analysis. AIAA 2011-3808, June 2011. 
${ }^{24}$ Nayani, Sudheer N.; and Campbell, Richard L.: Evaluation of Grid Modification Methods for On- and Off-Track Sonic Boom Analysis, AIAA 2013-0798, January 2013. 